\title{
Perturbative Unitarity Of Lee-Wick Quantum Field Theory
}

\author{
Damiano Anselmi ${ }^{1}$ and Marco Piva ${ }^{2}$ \\ Dipartimento di Fisica "Enrico Fermi", Università di Pisa, \\ Largo B. Pontecorvo 3, 56127 Pisa, Italy \\ and INFN, Sezione di Pisa, \\ Largo B. Pontecorvo 3, 56127 Pisa, Italy
}

\begin{abstract}
We study the perturbative unitarity of the Lee-Wick models, formulated as nonanalytically Wick rotated Euclidean theories. The complex energy plane is divided into disconnected regions and the values of a loop integral in the various regions are related to one another by a nonanalytic procedure. We show that the one-loop diagrams satisfy the expected, unitary cutting equations in each region: only the physical degrees of freedom propagate through the cuts. The goal can be achieved by working in suitable subsets of each region and proving that the cutting equations can be analytically continued as a whole. We make explicit calculations in the cases of the bubble and triangle diagrams and address the generality of our approach. We also show that the same higher-derivative models violate unitarity if they are formulated directly in Minkowski spacetime.
\end{abstract}

\footnotetext{
${ }^{1}$ damiano.anselmi@unipi.it

${ }^{2}$ marco.piva@df.unipi.it
} 


\section{Introduction}

The nonrenormalizability of the Hilbert-Einstein Lagrangian [1] teaches us that, if we want to solve the problem of quantum gravity, we have to explore new sectors of quantum field theory and maybe relax some assumptions we are accustomed to. In this respect, an interesting subsector of quantum field theory is represented by the local, higher-derivative theories, because there is still a possibility that the search for a consistent theory of quantum gravity might lead there.

However, the formulation of higher-derivative theories turns out to be less trivial than expected. For example, when the free propagators have complex poles, the theories cannot be consistently defined in Minkowski spacetime [2], in general, because they generate nonlocal, non-Hermitian divergences, which cannot be subtracted away without destroying the nature of the theory itself.

The Lee-Wick (LW) models [3] are a special subclass of local, higher-derivative theories, which have the possibility of reconciling renormalizability and unitarity. The propagators contain complex conjugate pairs of extra poles, which we call $L W$ poles, besides the poles corresponding to the physical degrees of freedom and the degrees of freedom introduced by the gauge fixing (which are those propagated by the temporal and the longitudinal components of the gauge fields, as well as the Faddeev-Popov ghosts). The Lee-Wick models are claimed to lead to a perturbatively unitary $S$ matrix $[3,4,5]$. Because of their unusual features, their formulation has been the object of several investigations. Like all higher-derivative theories, they violate microcausality. Nakanishi [6] showed that if the loop space momenta are integrated on their natural, real values, as Lee initially seemed to suggest [7], Lorentz invariance is violated. Cutkosky et al. (CLOP) showed [5] that the $S$ matrix is not analytic when pairs of LW poles pinch the integration path of the energy. They proposed to treat such a pinching, which we call $L W$ pinching, by means of a limiting procedure, known as CLOP prescription. Among other things, the CLOP prescription removes the problems found by Nakanishi. In simple diagrams, it gives an unambiguous, Lorentz invariant and unitary result, as confirmed by the calculations of Grinstein et al. [8]. However, it seems a bit artificial, since it cannot be incorporated into a Lagrangian and ambiguities are still present. For a while it was thought that such ambiguities survived only at high orders diagrams [5], but recently it has been shown that they are present also at one loop [9].

These pieces of information need to be clarified and properly assessed. To answer some of the open questions, a new formulation of the Lee-Wick models has been recently proposed [9], by viewing them as nonanalytically Wick rotated Euclidean higher-derivative theories.

Since the Minkowski formulation is not viable [2], we have no choice but start from the 
Euclidean version of the higher-derivative theories. However, the Wick rotation turns out to be nonanalytic, because of the LW pinching, to the extent that the complex energy plane is divided into disjoint regions $\mathcal{A}_{i}$ of analyticity. The Lorentz violation is avoided by working in a generic Lorentz frame, with generic external momenta, and deforming the integration domain of the space loop momenta to complex values in a suitable way. It turns out that the models are intrinsically equipped with all that is necessary to define them properly. There is no need of the CLOP prescription, or any other prescription, to handle the pinching of the LW poles. Moreover, the CLOP prescription leads to physical results that are ambiguous, even in a simple case such as the bubble diagram with different physical masses [9]. Therefore, the ad hoc prescriptions should be dropped.

Because the Lee-Wick models have been reformulated anew, and the new formulation leads to predictions that are quantitatively different from those of the previous approaches, it is compulsory to investigate perturbative unitarity in the new formulation. Writing the $S$ matrix as $S=1+i T$, the unitarity relation $S S^{\dagger}=1$ is equivalent to $T-T^{\dagger}=i T T^{\dagger}$. This identity can be expressed diagrammatically by means of the so-called cutting equations [10], which relate the discontinuity of an amplitude to the sum of cut diagrams (see also [11] for a recent extension and [12] for an algebraic reformulation). The cut diagrams are built with cut propagators and shadowed vertices, in addition to the usual propagators and vertices. In this paper, we study the cutting equations in the one-loop bubble and triangle diagrams explicitly, but the procedure can be extended to all the one-loop diagrams.

The cutting equations must be derived within the formulation of the models as nonanalytically Wick rotated Euclidean theories. To achieve this goal, we show that it is possible to derive the cutting equations in suitable subsets $\mathcal{O}_{i}$ of the analytic regions $\mathcal{A}_{i}$ and extend their validity to the whole $\mathcal{A}_{i}$ by analytic continuation. The analytic continuation of the cut diagrams is something that also requires some attention, because it is not discussed in the available literature.

The results we find confirm that the cutting equations of the LW models are consistent with perturbative unitarity. The contributions of the poles of each LW pair mutually cancel, so only the physical degrees of freedom propagate through the cuts.

Our findings also suggest that the cancellation mechanism, which is encoded in formula (5.6), is a general property of all diagrams. While the bubble diagram is too special to argue in favor of general properties, the derivation of the cutting equations for the triangle diagram is sufficiently general to be applied to all the one-loop diagrams. The generalization to diagrams with more loop is less direct, but it appears to be mostly a technical matter, which is why we believe that our results can be the starting point to derive a proof of perturbative unitarity to all orders. 
Finally, to emphasize the importance of the nonanalytic Wick rotation, we show that the same higher-derivative models do violate unitarity when they are defined directly in Minkowski spacetime.

The LW models are important not only theoretically, but also because they may have phenomenological applications. Among those that have been considered in the literature, we mention extensions of QED [4], physics beyond the standard model [13] and grand unified theories [14], as well as the search for a consistent theory of quantum gravity [15, 16]. In ref. [9] it was also noted that the unusual behaviors of the physical amplitudes, due to the violations of analyticity, may have important phenomenological consequences, for example allow us to measure some key physical constants of the LW models, such as the scales associated with the higher-derivative terms.

The paper is organized as follows. In section 2 we recall the formulation of the models. In section 3 we study the analytic continuation of the cut diagrams. In section 4 we reconsider the bubble diagram in standard theories and derive its cutting equations in a setting that is sufficiently general to ease out the extension to the LW models. In section 5 , we derive the cutting equations of the bubble diagram in the LW models and show that only the physical degrees of freedom propagate through the cuts. In section 6 , we do the same for the triangle diagram. In section 7, we extend our results to Feynman diagrams with nontrivial numerators and comment on the validity of our arguments in arbitrary diagrams. In section 8, we show that the higher-derivative theories of the LW class, if defined directly in Minkowski spacetime, do violate unitarity. Section 9 contains our conclusions.

\section{Lee-Wick models as nonanalytically Wick rotated Eu- clidean theories}

In this section we recall how the LW models are formulated as nonanalytically Wick rotated Euclidean theories [9]. For concreteness, it may be useful to have a specific theory in mind, such as the massive Lee-Wick $\varphi^{4}$ theory in four dimensions described by the Lagrangian

$$
\mathcal{L}=\frac{1}{2}\left(\partial_{\mu} \varphi\right)\left(1+\frac{\square^{2}}{M^{4}}\right)\left(\partial^{\mu} \varphi\right)-\frac{1}{2} m^{2} \varphi\left(1+\frac{\square^{2}}{M^{4}}\right) \varphi-\frac{\lambda}{4 !} \varphi^{4},
$$

whose free propagator reads in momentum space

$$
i D\left(p^{2}, m^{2}, \epsilon\right)=\frac{i M^{4}}{\left(p^{2}-m^{2}+i \epsilon\right)\left(\left(p^{2}\right)^{2}+M^{4}\right)} .
$$

More general propagators and more diverse theories can be considered, but they do not change the sense of the discussion that follows. In the limit $M \rightarrow \infty,(2.2)$ returns the standard 


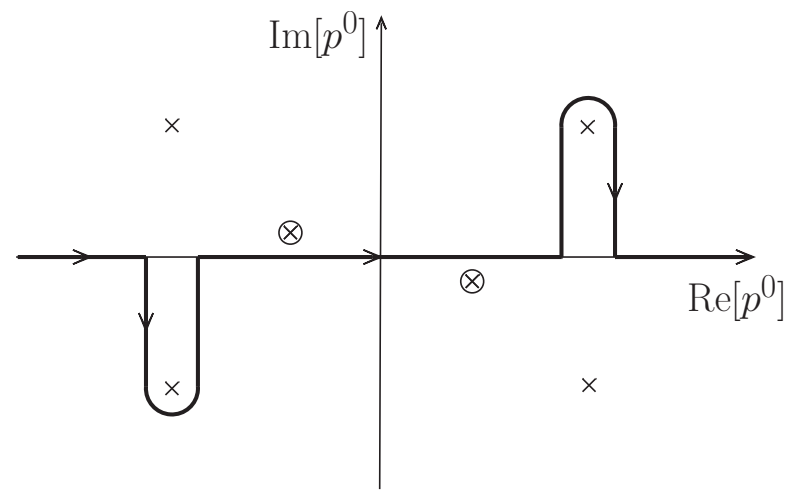

Figure 1: Integration path given by the Wick rotation

propagator, while at $M<\infty$ extra poles, which we call LW poles, are present besides the standard ones. The LW poles come in complex conjugate pairs, which we call $L W$ pairs.

The Wick rotation is simple for a single propagator. When the imaginary axis is rotated to the real one, we get the integration path of fig. 1, where the encircled crosses denote the standard poles and the non encircled crosses denote the LW poles. In generic Feynman diagrams, where more propagators are present, the Wick rotation is less trivial. Let us consider, for example, the bubble diagram, fig. 2. The loop integral is proportional to

$$
\mathcal{J}(p)=\int \frac{\mathrm{d}^{D} k}{(2 \pi)^{D}} D\left(k^{2}, m_{1}^{2}, \epsilon_{1}\right) D\left((p-k)^{2}, m_{2}^{2}, \epsilon_{2}\right) .
$$

For the sake of generality, we take different masses $m_{1}, m_{2}$ and different infinitesimal widths $\epsilon_{1}, \epsilon_{2}$. When we vary the external momentum $p$, the poles of the first propagator are fixed, while those of the second propagator move on the complex $k^{0}$ plane. Assuming for simplicity that the external space momentum $\mathbf{p}$ vanishes and taking $p^{0}$ real, the Wick rotation gives the integration path of fig. 3 .

We see that the left LW pair of a propagator is always above the integration path, while the right LW pair is always below. This property holds in arbitrary diagrams. A pinching, which

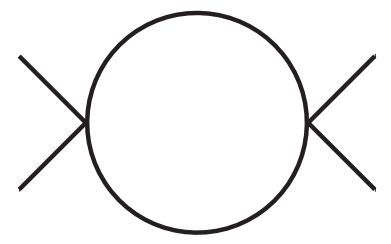

Figure 2: Bubble diagram 


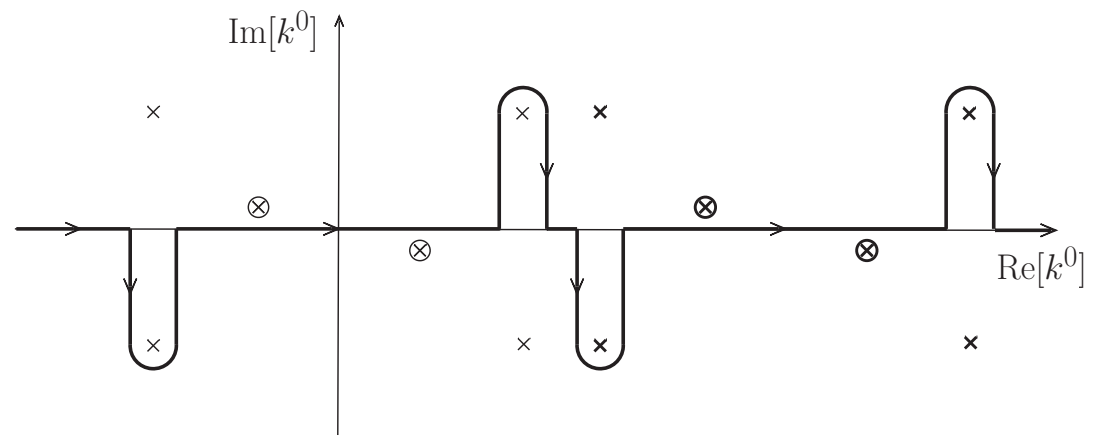

Figure 3: Integration path of the bubble diagram

we call $L W$ pinching, occurs when the left (right) LW pair of the first propagator hits the right (left) LW pair of the second propagator. The threshold of this pinching is $p^{2}=2 M^{2}$. With complex $p^{0}$, other types of LW pinchings occur: the bottom LW pole of the left LW pair of the first propagator can hit the top LW pole of the right LW pair of the second propagator, and so on. The thresholds of these pinchings are $p^{2}= \pm 4 i M^{2}$. Several such situations are symmetric to one another, so it suffices to study a single representative of each symmetric subset.

The threshold associated with a LW pinching will be called $L W$ threshold. We anticipate that the LW thresholds are not associated with discontinuities of the amplitudes, in agreement with unitarity. However, they are associated with nonanalytic behaviors of the amplitudes.

We focus on the pure LW pinching, which involves two LW poles, because at one loop it is the only LW pinching that has thresholds on the real axis. The mixed LW pinching, which occurs between a LW pole and a standard pole, needs complex external momenta $p$ and its thresholds are far away from the real axis.

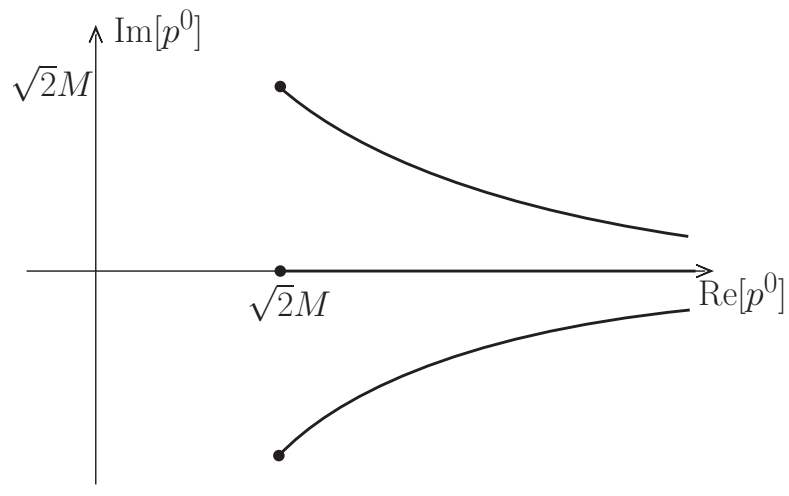

Figure 4: LW thresholds and LW branch cuts at $\mathbf{p}=0$ 


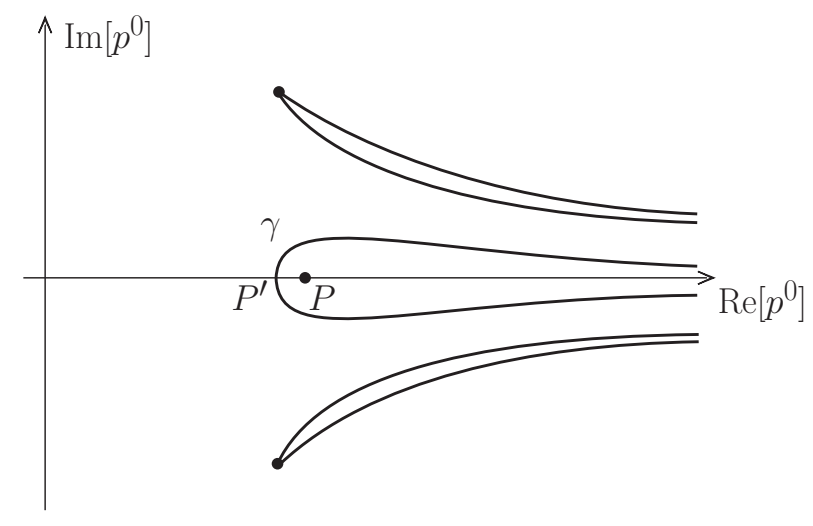

Figure 5: Areas of LW pinching at $\mathbf{p} \neq 0$

To evaluate $\mathcal{J}(p)$, we first integrate over the loop energy $k^{0}$ by means of the residue theorem, after which we remain with the integral on the loop space momentum $\mathbf{k}$. At $\mathbf{p}=0$, if we keep the $\mathbf{k}$ integration domain rigid, i.e. integrate $\mathbf{k}$ on its natural, real values, the positions of the LW thresholds and the LW branch cuts as functions of the external (complex) energy $p^{0}$ are those shown in fig. 4, plus their reflections with respect to the imaginary axis. In particular, the branch cut on the real axis is made by the solutions of the pinching condition

$$
p^{0}=\sqrt{\mathbf{k}^{2}+i M^{2}}+\sqrt{\mathbf{k}^{2}-i M^{2}} .
$$

When $p^{0}$ crosses one of the curves shown there, a pole of the integrand crosses the $\mathbf{k}$ integration domain. The cuts can be analytically deformed by deforming the $\mathbf{k}$ integration domain before it is crossed by the pole, so as to prevent the crossing from actually occurring.

Something interesting happens at nonvanishing, real $\mathbf{p}$. Keeping the $\mathbf{k}$ integration domain rigid again, we find that each cut of figure 4 enlarges into the regions $\tilde{\mathcal{A}}_{i}$ shown in fig. 5 . We denote the main region, i.e. the one that contains the imaginary axis, by $\tilde{\mathcal{A}}_{0}$. There, the Wick rotation is analytic, because no LW pinching occurs. The curve $\gamma$ is the boundary of a different region, which we denote by $\tilde{\mathcal{A}}_{P}$, which contains the positive real axis above the threshold $p^{2}=2 M^{2}$, located in the point $P$. The points of $\tilde{\mathcal{A}}_{P}$ are the solutions of the pinching condition

$$
p^{0}=\sqrt{\mathbf{k}^{2}+i M^{2}}+\sqrt{(\mathbf{k}-\mathbf{p})^{2}-i M^{2}}
$$

Note that $\gamma$ does not intersect the real axis in $P$, but in another point $P^{\prime}$, located below the threshold. Working out the coordinates of $P$ and $P^{\prime}$, we find

$$
P: \quad p^{0}=\sqrt{2 M^{2}+\mathbf{p}^{2}} \equiv E_{P}, \quad P^{\prime}: \quad p^{0}=\sqrt{\frac{\mathbf{p}^{2}}{2}+\sqrt{\frac{\left(\mathbf{p}^{2}\right)^{2}}{4}+4 M^{4}}} \equiv E_{P^{\prime}}
$$


which satisfy $\sqrt{2} M<E_{P^{\prime}}<E_{P}, E_{P}-E_{P^{\prime}}<\sqrt{2} M$.

Since the location of $P^{\prime}$ has no Lorentz invariant meaning, Lorentz invariance appears to be violated. Recall that fig. 5 is derived by keeping the loop space momentum $\mathbf{k}$ real. However, the integration path of fig. 1 shows that the loop energy is not everywhere real, so Lorentz invariance implies the loop momentum cannot be everywhere real. To recover Lorentz invariance, the $\mathbf{k}$ integration domain must be deformed to include complex values. Moreover, the deformation must turn the surfaces of fig. 5 into Lorentz invariant lines (i.e. solutions of Lorentz invariant conditions), similar to those of fig. 4. In particular, it must turn the region $\tilde{\mathcal{A}}_{P}$ into the half line of the real axis that goes from the point $P$ to $+\infty$, which we denote by $\mathcal{O}_{P}$. Indeed, $\mathcal{O}_{P}$ is Lorentz invariant, while any extended region is not.

It can be argued [9] that the domain deformation just described restores both Lorentz invariance and analyticity above the LW threshold. To give more details on this, let us write the propagator (2.2) in the equivalent form

$$
i D_{0}\left(p^{2}, m^{2}, \epsilon\right)+i D_{\mathrm{LW}}\left(p^{2}, m^{2}\right)
$$

where

$$
D_{0}\left(p^{2}, m^{2}, \epsilon\right)=\frac{M^{4}}{M^{4}+m^{4}} \frac{1}{p^{2}-m^{2}+i \epsilon}, \quad D_{\mathrm{LW}}\left(p^{2}, m^{2}\right)=-\frac{M^{4}}{M^{4}+m^{4}} \frac{p^{2}+m^{2}}{\left(p^{2}\right)^{2}+M^{4}} .
$$

We can use this decomposition to separate the contributions of the physical poles from the ones of the LW poles in every diagram. Then, we focus on the contributions that involve LW poles. For example, in the bubble diagram we take

$$
\mathcal{J}_{\mathrm{LW}}(p)=\int \frac{\mathrm{d}^{D} k}{(2 \pi)^{D}} D_{\mathrm{LW}}\left(k^{2}, m_{1}^{2}\right) D_{\mathrm{LW}}\left((k-p)^{2}, m_{2}^{2}\right) .
$$

The function $\mathcal{J}_{\mathrm{LW}}(p)$ is analytic and Lorentz invariant in the main region $\tilde{\mathcal{A}}_{0}$, because the Wick rotation is analytic there. In $\mathcal{O}_{P}$ (which means on the real axis above $P$ ) the domain deformation described above leads to the result [9]

$$
\mathcal{J}_{\mathrm{LW}}(p)=\frac{1}{2}\left[\mathcal{J}_{\mathrm{LW}}^{0+}(p)+\mathcal{J}_{\mathrm{LW}}^{0-}(p)\right]
$$

where the functions $\mathcal{J}_{\mathrm{LW}}^{0 \pm}(p)$ are obtained by analytically continuing $\mathcal{J}_{\mathrm{LW}}(p)$ from $\tilde{\mathcal{A}}_{0}$ to $\mathcal{O}_{P}$ from the half plane $\operatorname{Im}\left[p^{0}\right]>0$ or from the half plane $\operatorname{Im}\left[p^{0}\right]<0$, respectively, as illustrated in fig. 6. The continuations can be stretched to neighborhoods of $\mathcal{O}_{P}$ above $P$, to eventually cover an extended region $\mathcal{A}_{P}$ such as the one shown in fig. 7 .

In the end, the complex plane is divided into disjoint regions $\mathcal{A}_{i}$ of analyticy. We call $\mathcal{A}_{0}$ the analytic region that contains the imaginary axis. The function $\mathcal{J}_{\mathrm{LW}}(p)$ is analytic in each 


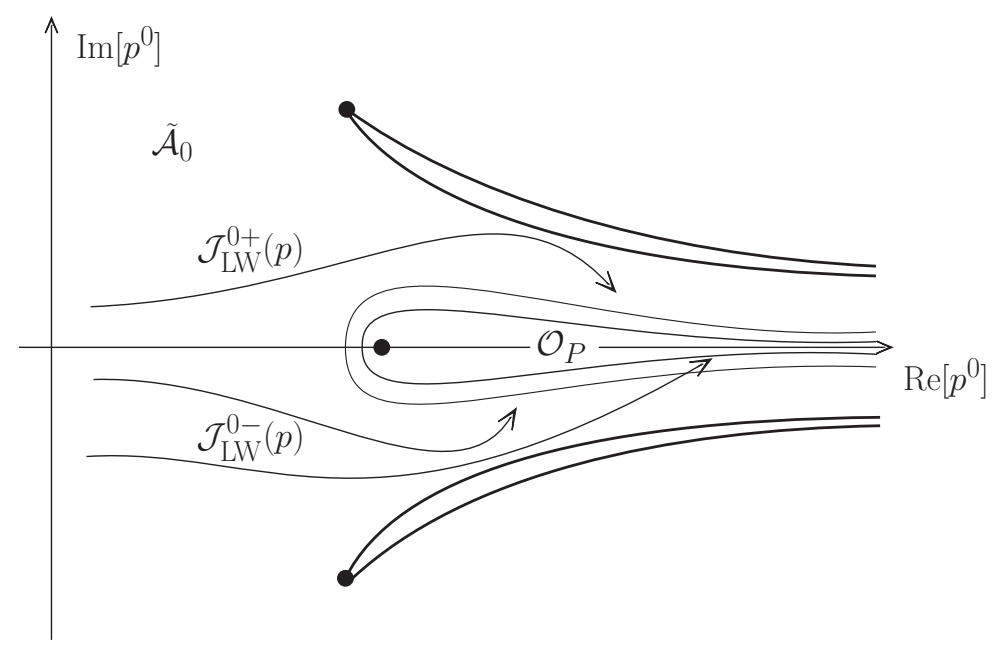

Figure 6: Definitions of $\mathcal{J}_{\mathrm{LW}}^{0+}(p)$ and $\mathcal{J}_{\mathrm{LW}}^{0-}(p)$

region, but not on the entire complex plane. Formula (2.8) relates the value of the function in $\mathcal{A}_{P}$ to the value of the function in $\mathcal{A}_{0}$. In particular, it ensures Lorentz invariance and analyticity in $\mathcal{A}_{P}$ thanks to the Lorentz invariance and analyticity in $\tilde{\mathcal{A}}_{0}$.

We stress again that the amplitudes must be evaluated at generic external momenta and in a generic Lorentz frame, because special Lorentz frames may squeeze some regions $\tilde{\mathcal{A}}_{i}$ into curves $\Gamma_{i}$. For example, in the center of mass frame $\mathbf{p}=0$, the region $\tilde{\mathcal{A}}_{P}$ of the bubble diagram is squeezed onto $\mathcal{O}_{P}$. The value of the amplitude in $\mathcal{O}_{P}$ is ill defined at $\mathbf{p}=0$, but can be worked out at $\mathbf{p} \neq 0$, where $\tilde{\mathcal{A}}_{P}$ is extended, by means of the deformation procedure explained above. Note that the deformation also squeezes $\tilde{\mathcal{A}}_{P}$ onto $\mathcal{O}_{P}$, but that happens when the amplitude

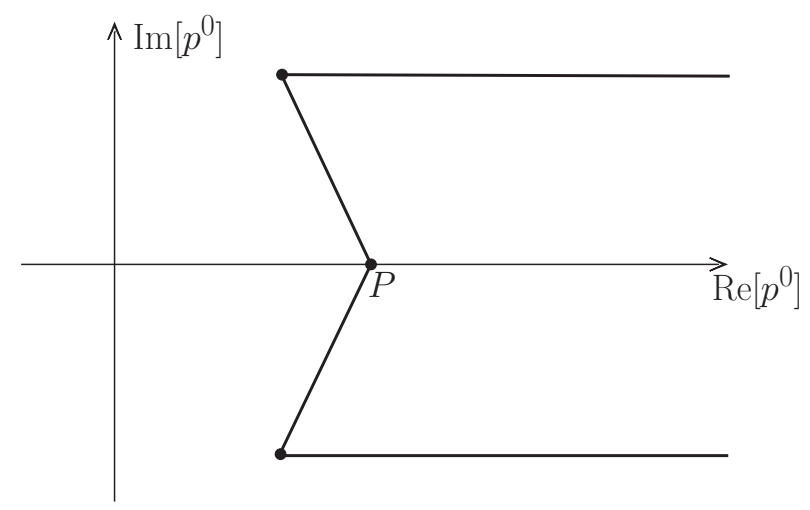

Figure 7: Analytic regions 
is evaluated inside of it, not before.

The integrand of $\mathcal{J}(p)$ is singular where the LW pinching occurs, but the singularity is integrable. Specifically, focus on the intersection $\mathcal{O}_{P}$ between $\tilde{\mathcal{A}}_{P}$ and the real axis. The pinching involves the left LW pair of one propagator and the right LW pair of the other propagator. For p small the integral around the pinching of the top LW poles is proportional to [9]

$$
\frac{\mathrm{d} \tau \mathrm{d} u}{\tau-i C|\mathbf{p}| u}
$$

where $C$ is a positive, $\mathbf{p}$-independent constant, $u=\cos \theta, \theta$ being the angle between the vectors $\mathbf{p}$ and $\mathbf{k}$, and $\tau$ parametrizes the fluctuation of $|\mathbf{k}|$ around the value it has at the singularity, which is $\sqrt{\left(p^{0}\right)^{4}-4 M^{4}} /\left(2\left|p^{0}\right|\right)$. The pinching of the bottom LW poles is described by flipping the sign in front of $i C$.

We see that, basically, a nonvanishing $|\mathbf{p}|$ provides the prescription for handling the integral. The limit $|\mathbf{p}| \rightarrow 0$ can be evaluated explicitly, because it squeezes $\tilde{\mathcal{A}}_{P}$ onto $\mathcal{O}_{P}$ bypassing the domain deformation. The result is

$$
\mathrm{d} \tau \mathrm{d} u\left[\mathcal{P} \frac{1}{\tau}+i \pi \operatorname{sgn}(u) \delta(\tau)\right] \rightarrow \mathrm{d} \tau \mathcal{P} \frac{1}{\tau}
$$

where $\mathcal{P}$ denotes the principal value and "sgn" is the sign function. In the last step we have performed the $u$ integration, which is trivial because the integrand of $\mathcal{J}(p)$ is $u$ independent at $|\mathbf{p}|=0$.

Let us describe what happens in more complicated diagrams. At one loop, the LW pinchings are similar to those of the bubble diagram. They are still described by fig. 3 and occur between the LW poles of any pair of propagators. As before, the LW thresholds on the real axis are given by the formula $p^{2}=2 M^{2}$, where now $p$ is any sum of external (incoming) momenta. In section 6 the triangle diagram is studied in detail. With more loops, the LW thresholds can involve both LW poles and physical poles. However, apart from minor differences, the arguments and properties outlined above - such as the recovery of analyticity and Lorentz invariance by means of the domain deformation, the behavior (2.9) of the integral around the potential singularity due to the LW pinching, as well as formula (2.8) - are still expected to hold, because their essential features are not related to the specific diagrams we have considered. More details on this can be found in section 7 .

\section{Analytic continuation of the cut diagrams}

In this section, we explain how to analytically continue the cutting equations for the study perturbative unitarity. Due to the domain deformation explained in the previous section, we 
have to include complex values of the loop space momenta k. Nevertheless, the contributions to the cutting equations due to the poles of the same LW pair still compensate each other. This result is ensured by the key formula (5.6). That formula only holds at $\epsilon=0$, where standard regions of the complex plane are squeezed to the real axis (see below). We have to clarify how to work at nonzero $\epsilon$ and when exactly the limit $\epsilon \rightarrow 0$ must be taken, if before or after the domain deformation.

We first discuss related issues in standard theories, then move to the LW models.

When $\epsilon \rightarrow 0$, the standard pinching takes place. Consider, for example, the cut version of the standard bubble diagram of a massive field of mass $m$. The branch points are $p^{0}= \pm 2 m$ and the cuts are $p^{0} \geqslant 2 m$ and $p^{0} \leqslant-2 m$, located on the real axis, where $p^{0}$ denotes the external energy and $\mathbf{p}$ is assumed to vanish. Those cuts are squeezed regions, one of which is shown in fig. $8(c)$. At nonvanishing $\epsilon$, each cut splits into two cuts, as shown in fig. $8(a)$, with branch points $p^{0}= \pm\left(2 m^{2}-i \epsilon\right) / m$ and $p^{0}= \pm\left(2 m^{2}+i \epsilon\right) / m$. Such cuts do not intersect the real axis at $\epsilon \neq 0$, so we are allowed to study the cutting equation in any interval of the real axis and analytically continue the result to the whole real axis.

The limit $\epsilon \rightarrow 0$ divides the complex plane into disconnected regions. If we deform the cuts analytically before the limit, we may obtain fig. $8(b)$, the disconnected regions being $\mathcal{A}$ and $\mathcal{A}^{\prime}$. However, what physics prescribes is fig. $8(c)$, where the region $\mathcal{A}^{\prime}$ is squeezed to the real axis. The value of the amplitude on the real axis is obtained by approaching the real axis from above, while the value of the complex conjugate amplitude is obtained by approaching the real axis from below. Let us inquire about the value of the amplitude in the region $\mathcal{A}^{\prime}$.

It is easy to show that, in the limit $\epsilon \rightarrow 0$, the value of the cut diagram in the intersection $\mathcal{A}_{\perp}^{\prime}$ between $\mathcal{A}^{\prime}$ and the real axis is equal to the discontinuity of the amplitude. Indeed, consider fig. 9 , which shows the complex energy plane in the case of $(i)$ the bubble diagram (fig. 2), (ii) its conjugate and (iii) minus the sum of the two cut diagrams (fig. 12), respectively. The cuts are displaced from the real axis at $\epsilon \neq 0$. In what follows we imagine to take $\epsilon \rightarrow 0$ and just report the values of the diagrams in this limit. The cutting equation tells us that the sum $(i)+(i i)$

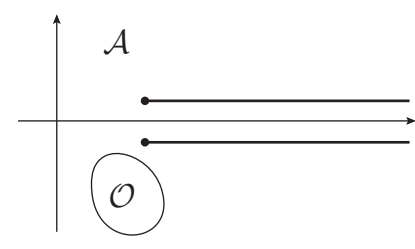

(a)

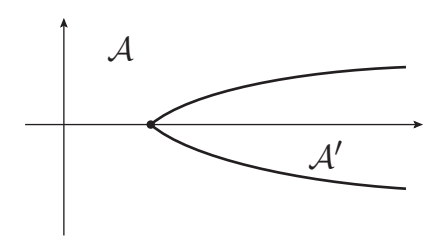

(b)

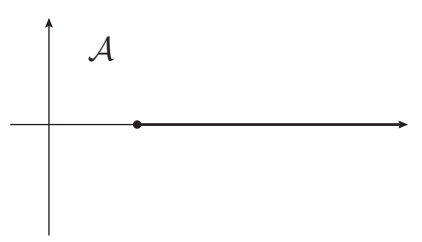

(c)

Figure 8: Analytic regions of the standard bubble diagram 


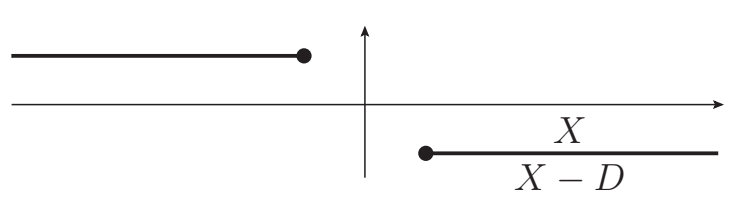

(i)

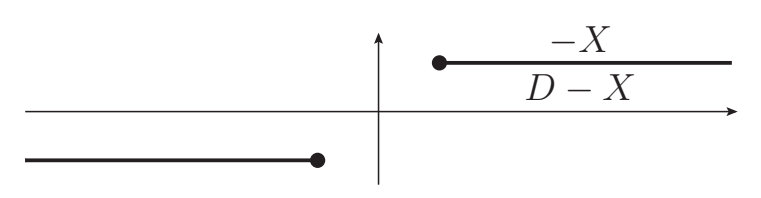

(ii)

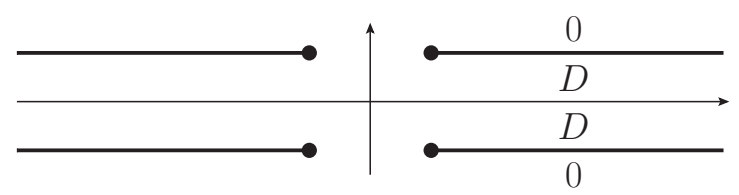

(iii)

Figure 9: Branch cuts of the cutting equations for the standard bubble diagram

is equal to $(\mathrm{iii})$ and that $(\mathrm{iii})$ vanishes everywhere except in the cuts for $\epsilon \rightarrow 0$. Let $X$ denote the value of the bubble diagram above the cut in that limit and $D=-1 /(8 \pi) \sqrt{1-\left(4 m^{2} / p^{2}\right)}$ the well-known value of the discontinuity. Then, the value of the bubble diagram below the cut is $X-D$. Since the sum $(i)+(i i)$ must vanish both above the two cuts and below them, we infer that the value of the conjugate bubble diagram is $-X$ above the cut and $D-X$ below the cut. This implies that the region in between the cuts of figure $(i i i)$ must have value $D$, which is what we claimed. Thus, the analytic function of the region $\mathcal{A}^{\prime}$ shown in fig. $8(b)$ is $-1 /(8 \pi) \sqrt{1-\left(4 m^{2} / p^{2}\right)}$, while the analytic function of the region $\mathcal{A}$ is identically zero.

We see that a single function that is analytic in a neighborhood of the real axis at $\epsilon \neq 0$ breaks into multiple analytic functions when $\epsilon$ tends to zero. Each cut diagram is analytic throughout the real axis at $\epsilon \neq 0$. Instead, at $\epsilon=0$ the real axis is divided into several domains and the cut diagram is separately analytic in each domain.

These remarks are useful when we move to the Lee-Wick models. We consider a generic oneloop diagram and describe how the cutting equations are derived in sections 5 and 6 , focusing on the regions that have intersections with the real axis. We must combine the discussion about the analytic regions associated with the LW pinching with the discussion about the usual pinching. The standard threshold reads $p^{2}=\left(m_{i}+m_{j}\right)^{2}$, where $m_{i}$ and $m_{j}$ are the masses of two particles circulating in the loop and $p$ is a sum of incoming momenta. The LW threshold on the real axis is $p^{2}=2 M^{2}$.

Assume first that $2 M^{2}>\left(m_{i}+m_{j}\right)^{2}$ and $P^{\prime}$ is located above $Q$. The cut diagram leads to a typical situation like the one of fig. 10. Above the LW threshold $P$, we study the difference $i \mathcal{M}-i \mathcal{M}^{*}$, where $\mathcal{M}=-i \lambda^{2} \mathcal{J} / 2$ is the amplitude, by working at $\epsilon \neq 0$ in a subdomain of $\tilde{\mathcal{A}}_{3}$, for example an interval $\mathcal{D}_{3}$ of the real axis. Then, we perform the domain deformation, which squeezes the region $\tilde{\mathcal{A}}_{3}$ to the real axis, till it becomes the portion $\mathcal{A}_{3}=\mathcal{O}_{P}$ of the real axis from 


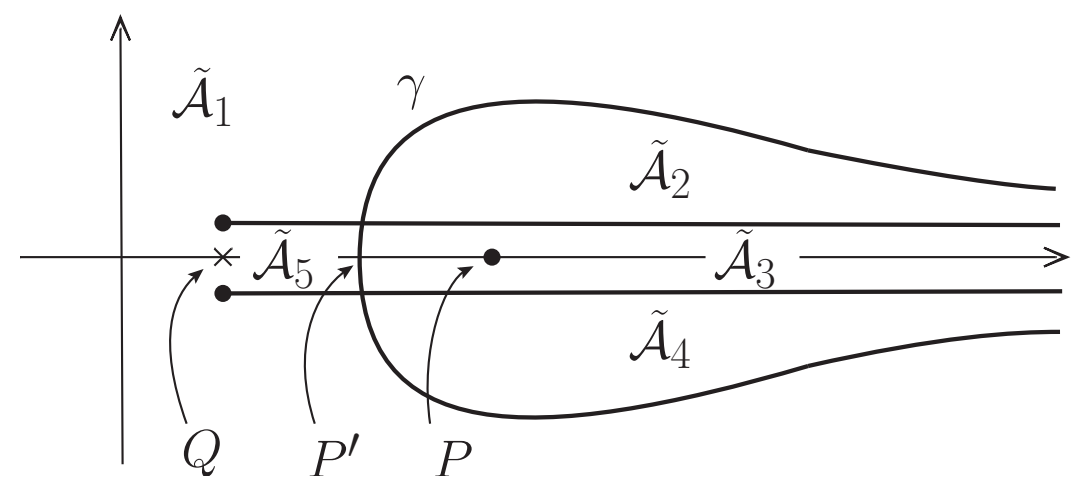

Figure 10: Standard pinching and LW pinching

$P$ to infinity. We show that the calculation can be extended through the domain deformation. Nevertheless, $i \mathcal{M}-i \mathcal{M}^{*}$ does not have the expected form compatible with unitarity, as long as $\epsilon$ remains nonzero. At the end, we take the limit $\epsilon \rightarrow 0$ and prove that $i \mathcal{M}-i \mathcal{M}^{*}$ can be expressed as predicted by the unitary cutting equation, encoded in the identity $i T-i T^{\dagger}=-T T^{\dagger}$.

Below the threshold $P$, the domain deformation is unnecessary. We split the calculation in two parts. The limit $\epsilon \rightarrow 0$ makes two standard poles coincide in $Q$ and divides the positive real axis below $P^{\prime}$ into two portions: one portion is the domain $\mathcal{D}_{1}$ that goes from the origin to $Q$, which belongs to the region $\tilde{\mathcal{A}}_{1}$; the other portion is the domain $\mathcal{D}_{5}$ that goes from $Q$ to $P^{\prime}$, which belongs to the region $\tilde{\mathcal{A}}_{5}$.

We can prove unitarity in $\mathcal{A}_{1}$ by working in an interval of $\mathcal{D}_{1}$, integrating rigidly on the loop space momenta, then taking $\epsilon \rightarrow 0$ and analytically continuing the cutting equation to the whole $\mathcal{D}_{1}$ and then $\mathcal{A}_{1}$.

Similarly, we can study $i \mathcal{M}-i \mathcal{M}^{*}$ in an interval of $\mathcal{D}_{5}$ (where we are allowed to rigidly integrate on the loop space momenta, since $\mathcal{D}_{5}$ is below $\gamma$ ) and then take the limit $\epsilon \rightarrow 0$. After that, we analytically continue the cutting equation to the interval of the real axis that goes from $Q$ to $P$. So doing, we cover the whole region $\mathcal{A}_{5}$, which is the segment of the real axis going from $Q$ to $P$, including the portion where we cannot integrate on the loop space

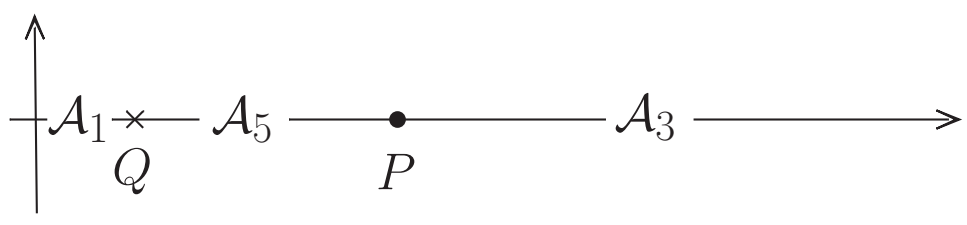

Figure 11: Intersections of the analytic regions with the real axis 
momenta rigidly. The analytic regions that intersect the real axis are then those of fig. 11.

Now, assume that $2 M^{2}>\left(m_{i}+m_{j}\right)^{2}$, but $P^{\prime}$ is located below $Q$. In that case, it seems that we cannot treat the portion of the real axis included from $Q$ to $P$ with the method explained above. Nevertheless, it is always possible to switch to a situation like the one of fig. 10. Indeed, for $\mathbf{p}=0, P^{\prime}$ coincides with $P$ and $Q$ is below $P^{\prime}=P$. By continuity, for nonvanishing, but sufficiently small p, $Q$ is still below $P^{\prime}$. This proves that there exists an open domain $\mathcal{O}_{5} \subset \tilde{\mathcal{A}}_{5}$ of the space of the external momenta where the point $Q$ is located below $P^{\prime}$. From $\mathcal{O}_{5}$ we can proceed as explained above and reach $\mathcal{A}_{5}$ after the analytic continuation.

Finally, when $2 M^{2}<\left(m_{i}+m_{j}\right)^{2}$ the point $Q$ is located above $P$. Then, below $Q$ we can proceed as in the pure LW case, while above $Q$ we can perform the domain deformation and let $\epsilon$ tend to zero at the end.

\section{The standard bubble diagram revisited}

In this section, we reconsider the standard bubble diagram and study its discontinuity. We generalize the usual derivation [17] in various directions, to prepare the extension to the LeeWick models.

We use the dimensional regularization and work in a generic Lorentz frame, instead of choosing, say, the external momentum $p=\left(p^{0}, \mathbf{p}\right)$ of the form $\left(p^{0}, 0\right)$. One reason is that this choice is only allowed for timelike external momenta. More importantly, we have seen that in the LW models it is crucial to keep the external space momentum $\mathbf{p}$ different from zero, to enlarge the region $\tilde{\mathcal{A}}_{P}$ of the complex plane, which is otherwise squeezed on the real axis.

We also take different masses $m_{1}, m_{2}$, and independent infinitesimal widths $\epsilon_{1}$, $\epsilon_{2}$, which we keep nonvanishing as long as we can. As shown in ref. [12], it is possible to work out more general versions of the cutting equations at $\epsilon \neq 0$ in the standard case. We will see in the next sections that this is no longer true in the LW case.

The loop integral reads

$$
i \mathcal{M}(p)=\frac{\lambda^{2}}{2} \int \frac{\mathrm{d}^{D} k}{(2 \pi)^{D}} \frac{1}{k^{2}-m_{1}^{2}+i \epsilon_{1}} \frac{1}{(k-p)^{2}-m_{2}^{2}+i \epsilon_{2}},
$$

where $\mathcal{M}(p)$ is the amplitude. We can equivalently write (4.1) as

$$
i \mathcal{M}(p)=\frac{\lambda^{2}}{2} \int \frac{\mathrm{d} k^{0} \mathrm{~d}^{D-1} \mathbf{k}}{(2 \pi)^{D}} \prod_{j=1}^{2} \frac{1}{\left(e_{j}-\omega_{j}+i \epsilon_{j}\right)\left(e_{j}+\omega_{j}-i \epsilon_{j}\right)},
$$

where $e_{1}=k^{0}, e_{2}=k^{0}-p^{0}, \omega_{1}=\sqrt{\mathbf{k}^{2}+m_{1}^{2}}$ and $\omega_{2}=\sqrt{(\mathbf{k}-\mathbf{p})^{2}+m_{2}^{2}}$. In going from (4.1) to (4.2), we have expanded the denominators for $\epsilon_{1}, \epsilon_{2}$ small and rescaled such widths. 
We perform the integral on $k^{0}$ by using the residue theorem and closing the integration path in the lower half $k^{0}$ plane. The relevant poles are located at $k^{0}=z_{1}$ and $k^{0}=z_{2}$, where

$$
z_{1}=\omega_{1}-i \epsilon_{1}, \quad z_{2}=p^{0}+\omega_{2}-i \epsilon_{2}
$$

The $k^{0}$ integral of $i \mathcal{M}$ leads to

$$
i \mathcal{M}(p)=-\frac{i \lambda^{2}}{2} \int \frac{\mathrm{d}^{D-1} \mathbf{k}}{(2 \pi)^{D-1}}\left[\operatorname{Res}\left(z_{1}\right)+\operatorname{Res}\left(z_{2}\right)\right]
$$

where $\operatorname{Res}(z)$ denotes the residue of the integrand (excluding the factor $\lambda^{2} / 2$ ) in $k^{0}=z$. We find

$$
\begin{aligned}
& \operatorname{Res}\left(z_{1}\right)=\frac{1}{2 z_{1}\left(z_{1}-z_{2}\right)\left(z_{1}+z_{2}-2 p^{0}\right)}=\frac{1}{2\left(\omega_{1}-i \epsilon_{1}\right) \Delta_{-}\left(\omega_{1}+\omega_{2}-p^{0}-i \epsilon_{+}\right)} \equiv r_{1}, \\
& \operatorname{Res}\left(z_{2}\right)=-\frac{1}{2\left(z_{2}-p^{0}\right)\left(z_{1}-z_{2}\right)\left(z_{1}+z_{2}\right)}=-\frac{1}{2\left(\omega_{2}-i \epsilon_{2}\right) \Delta_{-}\left(\omega_{1}+\omega_{2}+p^{0}-i \epsilon_{+}\right)} \equiv r_{2},
\end{aligned}
$$

while

$$
\Delta_{-}=z_{1}-z_{2}=\omega_{1}-\omega_{2}-p^{0}-i \epsilon_{-}
$$

and $\epsilon_{ \pm}=\epsilon_{1} \pm \epsilon_{2}$. The denominator $\Delta_{-}$gives ambiguous distributions, since the sign of $\epsilon_{-}$ depends on the order with which we perform the limits $\epsilon_{1} \rightarrow 0$ and $\epsilon_{2} \rightarrow 0$. As shown in ref. [12], the ambiguity must actually cancel out. Indeed, it does disappear as soon as we take the sum of the two residues, which gives

$$
\begin{aligned}
\operatorname{Res}\left(z_{1}\right)+\operatorname{Res}\left(z_{2}\right) & =-\frac{1}{4 z_{1}\left(z_{2}-p^{0}\right)}\left(\frac{1}{z_{1}+z_{2}-2 p^{0}}+\frac{1}{z_{1}+z_{2}}\right) \\
& =-\frac{1}{4 \omega_{1} \omega_{2}}\left(\frac{1}{\omega_{1}+\omega_{2}-p^{0}-i \epsilon_{+}}+\frac{1}{\omega_{1}+\omega_{2}+p^{0}-i \epsilon_{+}}\right) .
\end{aligned}
$$

In the last line we sent $\epsilon_{1}$ and $\epsilon_{2}$ to zero in a couple of places where they are unimportant. For example, the factor $1 / z_{1}$ can be replaced by $1 / \omega_{1}$. It is not convenient to make this replacement directly in formulas (4.4), because of the presence of the ambiguous denominator (4.5). In the rest of the paper, we make similar replacements, when they are allowed, without further notice.

The discontinuity $\operatorname{Disc} \mathcal{M}=2 i \operatorname{Im} \mathcal{M}$ of the amplitude can now be evaluated from (4.3) by means of the identity

$$
\frac{1}{x \pm i \epsilon}=\mathcal{P}\left(\frac{1}{x}\right) \mp i \pi \delta(x)
$$

where $\mathcal{P}$ denotes the principal value. We find $\operatorname{Disc} \mathcal{M}=i \lambda^{2} \Upsilon / 2$, where

$$
\Upsilon(p) \equiv \int \frac{\mathrm{d}^{D-1} \mathbf{k}}{(2 \pi)^{D-1}} \frac{2 \pi}{\left(2 \omega_{1}\right)\left(2 \omega_{2}\right)}\left[\delta\left(p^{0}-\omega_{1}-\omega_{2}\right)+\delta\left(p^{0}+\omega_{1}+\omega_{2}\right)\right] .
$$



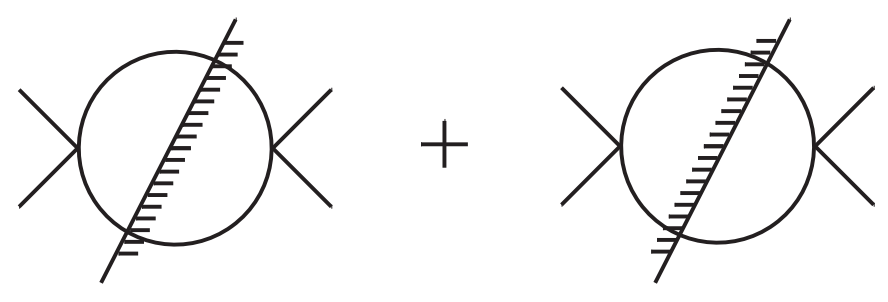

Figure 12: Cut bubble diagrams

If we relabel $q_{1}=k$ and $q_{2}=p-k$ and introduce integrals over $q_{1}$ and $q_{2}$, together with delta functions that impose $q_{i}^{0}= \pm \omega_{i}^{\prime}$, where $\omega_{i}^{\prime} \equiv \sqrt{\mathbf{q}_{i}^{2}+m_{i}^{2}}$, and $\mathbf{p}=\mathbf{q}_{1}+\mathbf{q}_{2}$, we can view $\delta\left(p^{0} \pm \omega_{1} \pm \omega_{2}\right)$ as the total energy conservation $\delta\left(p^{0}-q_{1}^{0}-q_{2}^{0}\right)$. Then we can write $\Upsilon$ as

$\int \frac{\mathrm{d}^{D} q_{1}}{(2 \pi)^{D}} \frac{\mathrm{d}^{D} q_{2}}{(2 \pi)^{D}}(2 \pi)^{D} \delta^{(D)}\left(p-q_{1}-q_{2}\right)\left[\frac{2 \pi \delta\left(q_{1}^{0}-\omega_{1}^{\prime}\right) 2 \pi \delta\left(q_{2}^{0}-\omega_{2}^{\prime}\right)}{\left(2 \omega_{1}^{\prime}\right)\left(2 \omega_{2}^{\prime}\right)}+\frac{2 \pi \delta\left(q_{1}^{0}+\omega_{1}^{\prime}\right) 2 \pi \delta\left(q_{2}^{0}+\omega_{2}^{\prime}\right)}{\left(2 \omega_{1}^{\prime}\right)\left(2 \omega_{2}^{\prime}\right)}\right]$ and finally

$$
\int \frac{\mathrm{d}^{D} q_{1}}{(2 \pi)^{D}} \frac{\mathrm{d}^{D} q_{2}}{(2 \pi)^{D}}(2 \pi)^{D} \delta^{(D)}\left(p-q_{1}-q_{2}\right)(2 \pi) \delta\left(q_{1}^{2}-m_{1}^{2}\right)(2 \pi) \delta\left(q_{2}^{2}-m_{2}^{2}\right)\left[\theta\left(q_{1}^{0}\right) \theta\left(q_{2}^{0}\right)+\theta\left(-q_{1}^{0}\right) \theta\left(-q_{2}^{0}\right)\right] .
$$

We see that Disc $\mathcal{M}=i \lambda^{2} \Upsilon / 2$ is equal to $i$ times the sum of the two cut diagrams $\mathcal{C}_{1}, \mathcal{C}_{2}$ shown in fig. 12 , i.e.

$$
i \mathcal{M}-i \mathcal{M}^{*}=-\frac{\lambda^{2}}{2} \Upsilon=-\mathcal{C}_{1}-\mathcal{C}_{2}
$$

The cut diagrams can be computed by replacing the ordinary propagators with the cut ones,

$$
\frac{i}{p^{2}-m^{2}+i \epsilon} \rightarrow 2 \pi \theta\left( \pm p^{0}\right) \delta\left(p^{2}-m^{2}\right)
$$

and equipping each shadowed vertex with a minus sign. The sign in front of $p^{0}$ is determined by the direction of the energy flow through the cut.

Formula (4.10) is nothing but the relation $i T-i T^{\dagger}=-T T^{\dagger}$ in the particular case we are considering and shows that the bubble diagram satisfies unitarity.

For completeness, we report the value of the integral $\Upsilon$ in four dimensions, which is well known:

$$
\Upsilon(p)=\frac{1}{8 \pi p^{2}} \theta\left(p^{2}-\left(m_{1}+m_{2}\right)^{2}\right) \sqrt{\left(p^{2}-m_{1}^{2}-m_{2}^{2}\right)^{2}-4 m_{1}^{2} m_{2}^{2}} .
$$

\subsection{A. Comments}

The procedure we have used in this section is general enough to be extended to the LW models. However, before moving to the LW case, we would like to emphasize the strategy of the calculation and compare it with other strategies. 
The first step has been to integrate on the energy by means of the residue theorem. Only after that, we have used the decomposition (4.7). The usage of that decomposition is extremely delicate, especially in products of distributions. For example, it is very inconvenient to use it before applying the residue theorem, directly in formula (4.1). If we do so (working at $\mathbf{p}=0$ and in the equal mass case $m_{1}=m_{2}=m$, for simplicity), we get

$$
\operatorname{Disc} \mathcal{M}=i \lambda^{2} \int \frac{\mathrm{d}^{D} k}{(2 \pi)^{D}}\left[\pi^{2} \delta\left(k^{2}-m^{2}\right) \delta\left((p-k)^{2}-m^{2}\right)-\mathcal{P} \frac{1}{k^{2}-m^{2}} \mathcal{P} \frac{1}{(k-p)^{2}-m^{2}}\right]
$$

The first contribution to (4.13), which can be rewritten as

$$
\frac{i \lambda^{2}}{4} \int \frac{\mathrm{d}^{D} q_{1}}{(2 \pi)^{D}} \frac{\mathrm{d}^{D} q_{2}}{(2 \pi)^{D}}(2 \pi)^{D} \delta^{(D)}\left(p-q_{1}-q_{2}\right)(2 \pi) \delta\left(q_{1}^{2}-m^{2}\right)(2 \pi) \delta\left(q_{2}^{2}-m^{2}\right),
$$

resembles the final result $i \lambda^{2} \Upsilon / 2$, with $\Upsilon$ given by (4.9). This lead some authors [16] to think that the two are equal and that the term with the two principal values in (4.13) does not contribute. Both statements are incorrect.

An apparent difference between $i \lambda^{2} \Upsilon / 2$ and (4.14) is that (4.9) contains the combination of theta functions $\Theta \equiv \theta\left(q_{1}^{0}\right) \theta\left(q_{2}^{0}\right)+\theta\left(-q_{1}^{0}\right) \theta\left(-q_{2}^{0}\right)$, while (4.14) does not. If we multiply the integrand of (4.14) by $1=\Theta+1-\Theta$, we can easily check that the difference $1-\Theta$, which is equal to $\theta\left(q_{1}^{0}\right) \theta\left(-q_{2}^{0}\right)+\theta\left(q_{1}^{0}\right) \theta\left(-q_{2}^{0}\right)$, gives zero. Thus, we can safely insert $\Theta$ in the integral of (4.14) and make it more similar to $i \lambda^{2} \Upsilon / 2$.

A more serious difference, instead, is the multiplying factor. Formula (4.14) is not really equal to $i \lambda^{2} / 2$ times (4.9), contrary to the claim of ref. [16], because it is multiplied by an additional factor $1 / 2$. The missing contribution must come from the product of the two principal values in formula (4.13). We have checked this fact numerically with a Mathematica program, starting from

$$
-i \lambda^{2} \int \frac{\mathrm{d}^{D} k}{(2 \pi)^{D}} \frac{k^{2}-m^{2}}{\left(k^{2}-m^{2}\right)^{2}+\epsilon^{2}} \frac{(p-k)^{2}-m^{2}}{\left((p-k)^{2}-m^{2}\right)^{2}+\epsilon^{2}}
$$

and taking smaller and smaller values of $\epsilon$. The argument used in ref. [16] to claim that this expression vanishes was to turn it to the Euclidean framework, where it naively becomes real, while in Minkowski spacetime it is purely imaginary. The point is that the Wick rotation is nontrivial in this case, because the integrand has poles in the first and third quadrants, which must be taken into account. More details can be found in ref. [2], where the problems of these types of Minkowski integrals are studied in depth. 


\section{The Lee-Wick bubble diagram}

In this section we study the LW version of the bubble diagram and show that it satisfies the correct cutting equation, with no propagation of unphysical degrees of freedom through the cuts. The loop integral is

$$
\begin{aligned}
i \mathcal{M} & =\frac{\lambda^{2}}{2} \int \frac{\mathrm{d}^{D} k}{(2 \pi)^{D}} D\left(k^{2}, m_{1}^{2}, \epsilon_{1}\right) D\left((k-p)^{2}, m_{2}^{2}, \epsilon_{2}\right) \\
& =\frac{\lambda^{2} M^{8}}{2} \int \frac{\mathrm{d} k^{0} \mathrm{~d}^{D-1} \mathbf{k}}{(2 \pi)^{D}} \prod_{j=1}^{2} \frac{1}{\left(e_{j}-\nu_{j}\right)\left(e_{j}+\nu_{j}\right)\left(e_{j}-\nu_{j}^{*}\right)\left(e_{j}+\nu_{j}^{*}\right)\left(e_{j}-\omega_{j}+i \epsilon_{j}\right)\left(e_{j}+\omega_{j}-i \epsilon_{j}\right)},
\end{aligned}
$$

where $\nu_{1}=\sqrt{\mathbf{k}^{2}+i M^{2}}, \nu_{2}=\sqrt{(\mathbf{k}-\mathbf{p})^{2}+i M^{2}}$ and the other definitions coincide with those of the previous section. For the reasons explained in section 2 , it is important to work at $\mathbf{p} \neq 0$.

Making the Wick rotation and closing the $k^{0}$ integration path in the lower half plane, fig. 3 tells us that we need the poles

$$
z_{1}=\omega_{1}-i \epsilon_{1}, \quad z_{2}=p^{0}+\omega_{2}-i \epsilon_{2}, \quad w_{1}=\nu_{1}, \quad w_{2}=p^{0}+\nu_{2},
$$

together with the conjugates $w_{1}^{*}$ and $w_{2}^{*}$. We find

$$
i \mathcal{M}=-\frac{i \lambda^{2}}{2} \int \frac{\mathrm{d}^{D-1} \mathbf{k}}{(2 \pi)^{D-1}}\left[\operatorname{Res}\left(z_{1}\right)+\operatorname{Res}\left(z_{2}\right)+\operatorname{Res}\left(w_{1}\right)+\operatorname{Res}\left(w_{2}\right)+\operatorname{Res}\left(w_{1}^{*}\right)+\operatorname{Res}\left(w_{2}^{*}\right)\right]
$$

We perform the domain deformations associated with the contributions of $\operatorname{Res}\left(w_{i}\right)$ and $\operatorname{Res}\left(w_{i}^{*}\right)$ in complex conjugate ways. Then, calling $U$ and $U^{*}$ the deformed domains, such contributions read

$$
-\frac{i \lambda^{2}}{2} \int_{U} \frac{\mathrm{d}^{D-1} \mathbf{k}}{(2 \pi)^{D-1}} \operatorname{Res}\left(w_{i}\right)-\frac{i \lambda^{2}}{2} \int_{U^{*}} \frac{\mathrm{d}^{D-1} \mathbf{k}}{(2 \pi)^{D-1}} \operatorname{Res}\left(w_{i}^{*}\right) .
$$

The other contributions, due to $\operatorname{Res}\left(z_{i}\right)$, can be calculated with the natural real $\mathbf{k}$ integration domain.

Now, we can write

$$
\left[\int_{U^{*}} \frac{\mathrm{d}^{D-1} \mathbf{k}}{(2 \pi)^{D-1}} \operatorname{Res}\left(w_{i}^{*}\right)\right]^{*}=\int_{U} \frac{\mathrm{d}^{D-1} \mathbf{k}}{(2 \pi)^{D-1}}\left[\operatorname{Res}\left(w_{i}^{*}\right)\right]^{*},
$$

where it is understood that the complex conjugations in $\left[\operatorname{Res}\left(w_{i}^{*}\right)\right]^{*}$ do not act on $\mathbf{k}$. We prove the identity

$$
\left[\operatorname{Res}\left(w_{i}^{*}\right)\right]^{*}=\operatorname{Res}\left(w_{i}\right)
$$

at $\epsilon_{1}=\epsilon_{2}=0$, which allows us to turn (5.5) into

$$
\left[\int_{U^{*}} \frac{\mathrm{d}^{D-1} \mathbf{k}}{(2 \pi)^{D-1}} \operatorname{Res}\left(w_{i}^{*}\right)\right]^{*}=\int_{U} \frac{\mathrm{d}^{D-1} \mathbf{k}}{(2 \pi)^{D-1}} \operatorname{Res}\left(w_{i}\right) .
$$


Formula (5.6) expresses the compensation between the contributions of the poles of the same LW pair to the cutting equations. It is the key result to prove that only the physical degrees of freedom propagate through the cuts.

To derive (5.6), observe that when $\epsilon_{1}$ and $\epsilon_{2}$ tend to zero we have

$$
\operatorname{Res}\left(w_{1}\right)-\left[\operatorname{Res}\left(w_{1}^{*}\right)\right]^{*}=\frac{\pi M^{6}}{2\left(m_{1}^{2}-i M^{2}\right) \nu_{1}} \frac{\tilde{\delta}\left(\left(\nu_{1}-p^{0}\right)^{2}-\omega_{2}^{2}\right)}{\left(\left(\nu_{1}-p^{0}\right)^{2}-(\mathbf{k}-\mathbf{p})^{2}\right)^{2}+M^{4}},
$$

where

$$
\tilde{\delta}(z) \equiv \lim _{\epsilon \rightarrow 0} \Delta_{\epsilon}(z), \quad \Delta_{\epsilon}(z) \equiv \frac{1}{2 i \pi}\left(\frac{1}{z-i \epsilon}-\frac{1}{z+i \epsilon}\right),
$$

is the usual delta distribution extended to complex values, which means that it is equal to zero anywhere but on the real axis, where it is the ordinary delta function. The right-hand side of (5.8) collects the terms where the limits $\epsilon_{1}, \epsilon_{2} \rightarrow 0$ are nontrivial, which need to be studied in detail.

Now we show that $\tilde{\delta}(z)$ does not contribute to the integrals (5.4). The pinching condition for both $\operatorname{Res}\left(w_{1}\right)$ and $\left[\operatorname{Res}\left(w_{1}^{*}\right)\right]^{*}$ is $(2.4)$, i.e. $p^{0}=\nu_{1}+\nu_{2}^{*}$, where, again, the complex conjugation does not act on $\mathbf{k}$. On the other hand, the argument of $\tilde{\delta}$ vanishes for $p^{0}=\nu_{1} \pm \omega_{2}$. These conditions cannot hold at the same time, because $p^{0}=\nu_{1}+\nu_{2}^{*}=\nu_{1} \pm \omega_{2}$ implies $-i M^{2}=m_{2}^{2}$. This fact has important consequences. When $\epsilon$ tends to zero, the contributions to $\Delta_{\epsilon}(z)$ provide potential singularities $\sim 1 / z$, with $z=\left(\nu_{1}-p^{0}\right)^{2}-\omega_{2}^{2}$. However, such singularities are actually integrable, because $z$ is complex. Therefore, we have two potential singularities, those due to the LW pinching and those due to $1 / z$. Both are separately integrable and could only give trouble if they occurred at the same time. Since this is impossible, the two contributions to $\Delta_{\epsilon}(z)$ mutually cancel for $\epsilon \rightarrow 0$ and the right-hand side of (5.8) can be dropped.

Similar arguments can be applied to $\operatorname{Res}\left(w_{2}\right)-\left[\operatorname{Res}\left(w_{2}^{*}\right)\right]^{*}$. We conclude that identity (5.6) holds. Thanks to it, the second integral of (5.4) is the complex conjugate of the first integral, so (5.7) holds.

Since formula (5.6) is valid only at $\epsilon_{1}=\epsilon_{2}=0$, we have to explain when such widths must be sent to zero. We work in the intervals $\mathcal{D}_{1}, \mathcal{D}_{3}$ and $\mathcal{D}_{5}$ defined in section 3 . In $\mathcal{D}_{3}$ we perform the domain deformation at $\epsilon_{1}, \epsilon_{2} \neq 0$, for the contributions due to $\operatorname{Res}\left(w_{i}\right)$ and $\operatorname{Res}\left(w_{i}^{*}\right)$. Instead, we keep the $\mathbf{k}$ integration domain rigid for the other contributions, as well as for the calculations in $\mathcal{D}_{1}$ and $\mathcal{D}_{5}$. Then, by means of identities like (5.8) and the calculations reported below, we check that the expected cutting equation separately holds in $\mathcal{D}_{1}, \mathcal{D}_{3}$ and $\mathcal{D}_{5}$, up to corrections of the form $\Delta_{\epsilon}(z)$, which are killed by the limit $\epsilon \rightarrow 0$. From section 3 , we know that the cutting equation can be analytically extended from $\mathcal{D}_{1}, \mathcal{D}_{3}$ and $\mathcal{D}_{5}$ to the regions $\mathcal{A}_{1}, \mathcal{A}_{3}$ and $\mathcal{A}_{5}$, respectively. 
Taking the limit $\epsilon_{1}, \epsilon_{2} \rightarrow 0$ on the contributions of $\operatorname{Res}\left(w_{i}\right)$ and $\operatorname{Res}\left(w_{i}^{*}\right)$ to (5.3), but keeping $\epsilon_{1}, \epsilon_{2} \neq 0$ in the contributions due to $\operatorname{Res}\left(z_{1}\right)$ and $\operatorname{Res}\left(z_{2}\right)$, formulas (5.4) and (5.7) give

$$
i \mathcal{M}=-\frac{i \lambda^{2}}{2} \int \frac{\mathrm{d}^{D-1} \mathbf{k}}{(2 \pi)^{D-1}}\left[\operatorname{Res}\left(z_{1}\right)+\operatorname{Res}\left(z_{2}\right)\right]-i \lambda^{2} \operatorname{Re} \int_{U} \frac{\mathrm{d}^{D-1} \mathbf{k}}{(2 \pi)^{D-1}}\left[\operatorname{Res}\left(w_{1}\right)+\operatorname{Res}\left(w_{2}\right)\right] .
$$

The discontinuity of the amplitude is then

$$
\operatorname{Disc} \mathcal{M}=2 i \operatorname{Im} \mathcal{M}=-i \lambda^{2} \int \frac{\mathrm{d}^{D-1} \mathbf{k}}{(2 \pi)^{D-1}} \operatorname{Im}\left[\operatorname{Res}\left(z_{1}\right)+\operatorname{Res}\left(z_{2}\right)\right] .
$$

This result proves that, as anticipated, the LW poles do not contribute to the imaginary part of the amplitude.

Now we show that the amplitude obeys the correct cutting equation. We have

$$
\operatorname{Res}\left(z_{1}\right)=r_{1} h\left(z_{1}\right), \quad \operatorname{Res}\left(z_{2}\right)=r_{2} h\left(z_{2}\right),
$$

where $r_{1}$ and $r_{2}$ are defined in formula (4.4) and

$$
h\left(k^{0}\right)=\frac{M^{4}}{\left(k^{2}\right)^{2}+M^{4}} \frac{M^{4}}{\left((k-p)^{2}\right)^{2}+M^{4}} .
$$

We understand the dependence of $h$ on the other variables besides $k^{0}$, because they are not important for the discussion.

As before, the ill-defined distributions contained in $r_{1}$ and $r_{2}$ cancel out in the sum of $\operatorname{Res}\left(z_{1}\right)$ and $\operatorname{Res}\left(z_{2}\right)$. We have

$$
\operatorname{Res}\left(z_{1}\right)+\operatorname{Res}\left(z_{2}\right)=r_{1} h\left(z_{1}\right)+r_{2} h\left(z_{2}\right)=u\left(z_{1}, z_{2}\right) v\left(z_{1}, z_{2}\right)
$$

where

$$
\begin{aligned}
& u\left(z_{1}, z_{2}\right)=\frac{z_{1}\left(z_{2}-p^{0}\right)\left[h\left(z_{1}\right)-h\left(z_{2}\right)\right]+z_{2}\left(z_{2}-p^{0}\right) h\left(z_{1}\right)-z_{1}\left(z_{1}-p^{0}\right) h\left(z_{2}\right)}{4 p^{0}\left(z_{1}-z_{2}\right) z_{1}\left(z_{2}-p^{0}\right)}, \\
& v\left(z_{1}, z_{2}\right)=\frac{1}{z_{1}+z_{2}-2 p^{0}}-\frac{1}{z_{1}+z_{2}} .
\end{aligned}
$$

It is clear that $u\left(z_{1}, z_{2}\right)$ is regular, since the numerator vanishes when $z_{1}=z_{2}$. Note that $h(z)$ is real and nonvanishing for real $z$. Thus, we can replace $u\left(z_{1}, z_{2}\right)$ by $u\left(\omega_{1}, \omega_{2}+p^{0}\right)$. At this point, we just need to take the imaginary part of $v\left(z_{1}, z_{2}\right)$ by means of formula (4.7), which gives

$$
\begin{aligned}
\operatorname{Disc} \mathcal{M} & =-i \pi \lambda^{2} \int \frac{\mathrm{d}^{D-1} \mathbf{k}}{(2 \pi)^{D-1}} u\left(\omega_{1}, \omega_{2}+p^{0}\right)\left[\delta\left(\omega_{1}+\omega_{2}-p^{0}\right)-\delta\left(\omega_{1}+\omega_{2}+p^{0}\right)\right] \\
& =\frac{i \lambda^{2}}{2} \frac{M^{8}}{\left(M^{4}+m_{1}^{4}\right)\left(M^{4}+m_{2}^{4}\right)} \Upsilon,
\end{aligned}
$$


where $\Upsilon$ is the integral (4.8). The second line is obtained by noting that the delta functions that appear in the first line of (5.11) simplify the value of the function $u$ considerably and allow us to make the replacements

$$
u\left(\omega_{1}, \omega_{2}+p^{0}\right) \delta\left(\omega_{1}+\omega_{2} \pm p^{0}\right) \rightarrow \pm \frac{1}{4 \omega_{1} \omega_{2}} \frac{M^{8} \delta\left(\omega_{1}+\omega_{2} \pm p^{0}\right)}{\left(M^{4}+m_{1}^{4}\right)\left(M^{4}+m_{2}^{4}\right)} .
$$

Following the procedure that we used in the standard case, we relabel $q_{1}=k$ and $q_{2}=p-k$ and obtain that $\operatorname{Disc} \mathcal{M}$ is equal to $i \lambda^{2} / 2$ times

$$
\int \frac{\mathrm{d}^{D} q_{1}}{(2 \pi)^{D}} \frac{\mathrm{d}^{D} q_{2}}{(2 \pi)^{D}}(2 \pi)^{D} \delta^{(D)}\left(p-q_{1}-q_{2}\right) D_{c}\left(q_{1}^{2}, m_{1}^{2}\right) D_{c}\left(q_{2}^{2}, m_{2}^{2}\right)\left[\theta\left(q_{1}^{0}\right) \theta\left(q_{2}^{0}\right)+\theta\left(-q_{1}^{0}\right) \theta\left(-q_{2}^{0}\right)\right],
$$

where

$$
D_{c}\left(p^{2}, m^{2}\right)=2 \pi \delta\left(p^{2}-m^{2}\right) \frac{M^{4}}{M^{4}+m^{4}} .
$$

So doing, we have shown that (4.10) holds in each interval $\mathcal{D}_{1}, \mathcal{D}_{3}$ and $\mathcal{D}_{5}$ of the real axis, with the cut propagators $\theta\left( \pm p^{0}\right) D_{c}\left(p^{2}, m^{2}\right)$. Then, we analytically continue (4.10) to the regions $\mathcal{A}_{1}, \mathcal{A}_{3}$ and $\mathcal{A}_{5}$. Unitarity is verified, because the cut propagators $\theta\left( \pm p^{0}\right) D_{c}\left(p^{2}, m^{2}\right)$ just propagate the physical degrees of freedom.

\section{The LW triangle diagram}

In this section we prove that the triangle diagram (fig. 13) also satisfies the correct cutting equation. The loop integral reads

$$
\begin{aligned}
& i \mathcal{M}=\lambda^{3} \int \frac{\mathrm{d}^{D} k}{(2 \pi)^{D}} D\left(k^{2}, m_{1}^{2}, \epsilon_{1}\right) D\left((k-p)^{2}, m_{2}^{2}, \epsilon_{2}\right) D\left((k-q)^{2}, m_{3}^{2}, \epsilon_{3}\right) \\
&=\lambda^{3} M^{12} \int \frac{\mathrm{d} k^{0} \mathrm{~d}^{D-1} \mathbf{k}}{(2 \pi)^{D}} \prod_{j=1}^{3} \frac{1}{\left(e_{j}-\nu_{j}\right)\left(e_{j}+\nu_{j}\right)\left(e_{j}-\nu_{j}^{*}\right)\left(e_{j}+\nu_{j}^{*}\right)\left(e_{j}-\omega_{j}+i \epsilon_{j}\right)\left(e_{j}+\omega_{j}-i \epsilon_{j}\right)}
\end{aligned}
$$

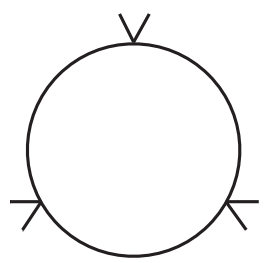

Figure 13: Triangle diagram 
where $e_{3}=k^{0}-q^{0}, \omega_{3}=\sqrt{(\mathbf{k}-\mathbf{q})^{2}+m_{3}^{2}}, \nu_{3}=\sqrt{(\mathbf{k}-\mathbf{q})^{2}+i M^{2}}$ and the other definitions coincide with those of the sections 4 and 5 .

Closing the $q^{0}$ integration path in the lower half plane, we just need the residues of the poles

$$
\begin{gathered}
z_{1}=\omega_{1}-i \epsilon_{1}, \quad z_{2}=p^{0}+\omega_{2}-i \epsilon_{2}, \quad z_{3}=q^{0}+\omega_{3}-i \epsilon_{3}, \\
w_{1}=\nu_{1}, \quad w_{2}=p^{0}+\nu_{2}, \quad w_{3}=q^{0}+\nu_{3},
\end{gathered}
$$

together with $w_{1}^{*}, w_{2}^{*}$ and $w_{3}^{*}$.

The LW thresholds that are located on the real axis are

$$
p^{2}=2 M^{2}, \quad q^{2}=2 M^{2}, \quad(p-q)^{2}=2 M^{2} .
$$

As explained in section 2, we must work at $\epsilon \neq 0$, choosing generic external momenta $p$ and $q$ in a generic Lorentz frame. In each region $\tilde{\mathcal{A}}_{i}$ of the space of $p$ and $q$, we choose one or more subdomains $\mathcal{O}_{i}$ with an accumulation point, typically intervals of the real axis. We separate the contributions where it is necessary to deform the integration domain of the loop space momentum $\mathbf{k}$ from the contributions where the deformation is unnecessary. It can be easily checked that an identity of the form (5.8) still holds. Like before, the right-hand side of (5.8) can be dropped, because the potential singularities $\sim 1 / z$ are integrable and do not occur simultaneously with the LW pinching. This leads again to the crucial cancellation formula (5.6), which ensures that only the standard residues contribute to the imaginary part of $\mathcal{M}$, in the limit $\epsilon \rightarrow 0$. At the end, we analytically continue the cutting equation from the subdomains $\mathcal{O}_{i}$ to the whole analytic regions $\mathcal{A}_{i}$.

Some attention must be paid to the ill-defined distributions, which are more tricky than in the previous case.

\section{A. The ill-defined distributions cancel out again}

The method we use here to prove this result is simpler than the one of the previous section, but we have to take the limit $\epsilon \rightarrow 0$ at a slightly earlier stage.

The residue of the integrand (excluding the factor $\lambda^{3}$ ) in $z_{1}$ is

$$
\begin{aligned}
\frac{M^{12}}{2 \omega_{1}\left(m_{1}^{4}+M^{4}\right)} \frac{1}{\left|\left(\omega_{1}-p^{0}\right)^{2}-\nu_{2}^{2}\right|^{2}\left|\left(\omega_{1}-q^{0}\right)^{2}-\nu_{3}^{2}\right|^{2}} \frac{1}{\left(\omega_{1}+\omega_{2}-p^{0}-i \epsilon_{+}^{12}\right)\left(\omega_{1}+\omega_{3}-q^{0}-i \epsilon_{+}^{13}\right)} \\
\quad \times \frac{1}{\left(\omega_{1}-\omega_{2}-p^{0}-i \epsilon_{-}^{12}\right)\left(\omega_{1}-\omega_{3}-q^{0}-i \epsilon_{-}^{13}\right)},
\end{aligned}
$$


where $\epsilon_{ \pm}^{i j} \equiv \epsilon_{i} \pm \epsilon_{j}$. The last two ratios are ill-defined distributions. We want to show that their contributions drop out. When $\epsilon \rightarrow 0$, the identity

$$
\frac{1}{\omega_{1}-\omega_{2}-p^{0}-i \epsilon_{-}^{12}}=\mathcal{P} \frac{1}{\omega_{1}-\omega_{2}-p^{0}}+i \pi \operatorname{sgn}\left(\epsilon_{-}^{12}\right) \delta\left(\omega_{1}-\omega_{2}-p^{0}\right),
$$

tells us that the ill-defined part is the one proportional to $\operatorname{sgn}\left(\epsilon_{-}^{12}\right)$. It is easy to check that in the expression $(6.1) \operatorname{sgn}\left(\epsilon_{-}^{12}\right)$ multiplies

$$
\begin{aligned}
& \frac{i \pi M^{12}}{4 \omega_{1} \omega_{2}\left(m_{1}^{4}+M^{4}\right)\left(m_{2}^{4}+M^{4}\right)} \frac{1}{\left|\left(\omega_{1}-q^{0}\right)^{2}-\nu_{3}^{2}\right|^{2}} \\
& \quad \times \frac{1}{\left(\omega_{1}+\omega_{3}-q^{0}-i \epsilon_{+}^{13}\right)} \frac{1}{\left(\omega_{1}-\omega_{3}-q^{0}-i \epsilon_{-}^{13}\right)} \delta\left(\omega_{1}-\omega_{2}-p^{0}\right)
\end{aligned}
$$

and cancels an analogous contribution coming from $\operatorname{Res}\left(z_{2}\right)$, which can be obtained by exchanging the poles $z_{1}$ and $z_{2}$, i.e. $\omega_{1}$ with $\omega_{2}+p^{0}$, as well as $\epsilon_{1}$ with $\epsilon_{2}$. Since (6.2) is invariant under these operations, but $\operatorname{sign}\left(\epsilon_{-}^{12}\right)$ turns into its opposite, the total vanishes.

A similar contribution to $\operatorname{Res}\left(z_{1}\right)$, proportional to $\operatorname{sgn}\left(\epsilon_{-}^{13}\right)$ cancels a contribution due to $\operatorname{Res}\left(z_{3}\right)$. Formula (6.1) also contains a term equal to $\operatorname{sgn}\left(\epsilon_{-}^{12}\right) \operatorname{sgn}\left(\epsilon_{-}^{13}\right)$ times

$$
-\frac{\pi^{2} M^{12} \delta\left(\omega_{1}-\omega_{2}-p^{0}\right) \delta\left(\omega_{1}-\omega_{3}-q^{0}\right)}{8 \omega_{1} \omega_{2} \omega_{3}\left(m_{1}^{4}+M^{4}\right)\left(m_{2}^{4}+M^{4}\right)\left(m_{3}^{4}+M^{4}\right)} .
$$

Summing the contributions of this type due to the three standard residues $z_{1}, z_{2}, z_{3}$, and noting that

$$
\operatorname{sgn}\left(\epsilon_{-}^{12}\right) \operatorname{sgn}\left(\epsilon_{-}^{13}\right)+\operatorname{sgn}\left(\epsilon_{-}^{23}\right) \operatorname{sgn}\left(\epsilon_{-}^{21}\right)+\operatorname{sgn}\left(\epsilon_{-}^{31}\right) \operatorname{sgn}\left(\epsilon_{-}^{32}\right)=1,
$$

which is easy to prove by choosing $\epsilon_{1}>\epsilon_{2}>\epsilon_{3}$, the total is (6.3), which has no imaginary part. We obtain a purely imaginary contribution to $i \mathcal{M}$ (an $i$ factor being brought by the residue theorem). The contributions of this type drop out of the cutting equation, whose left-hand side $i \mathcal{M}-i \mathcal{M}^{*}$ is manifestly real, if we manage to write the right-hand side in a manifestly real form.

\section{B. Unitarity}

Collecting the results found so far, we get

$$
i \mathcal{M}-i \mathcal{M}^{*}=2 \lambda^{3} \int \frac{\mathrm{d}^{D-1} \mathbf{k}}{(2 \pi)^{D-1}} \operatorname{Im}\left[\operatorname{Res}\left(z_{1}\right)+\operatorname{Res}\left(z_{2}\right)+\operatorname{Res}\left(z_{3}\right)\right] .
$$

Dropping the ill-defined distributions, $\operatorname{Res}\left(z_{1}\right)$ effectively simplifies to

$$
\begin{gathered}
\frac{M^{12}}{2 \omega_{1}\left(m_{1}^{4}+M^{4}\right)} \frac{1}{\left|\left(\omega_{1}-p^{0}\right)^{2}-\nu_{2}^{2}\right|^{2}\left|\left(\omega_{1}-q^{0}\right)^{2}-\nu_{3}^{2}\right|^{2}} \mathcal{P} \frac{1}{\omega_{1}-\omega_{2}-p^{0}} \mathcal{P} \frac{1}{\omega_{1}-\omega_{3}-q^{0}} \\
\times \frac{1}{\left(\omega_{1}+\omega_{2}-p^{0}-i \epsilon_{+}^{12}\right)\left(\omega_{1}+\omega_{3}-q^{0}-i \epsilon_{+}^{13}\right)} .
\end{gathered}
$$



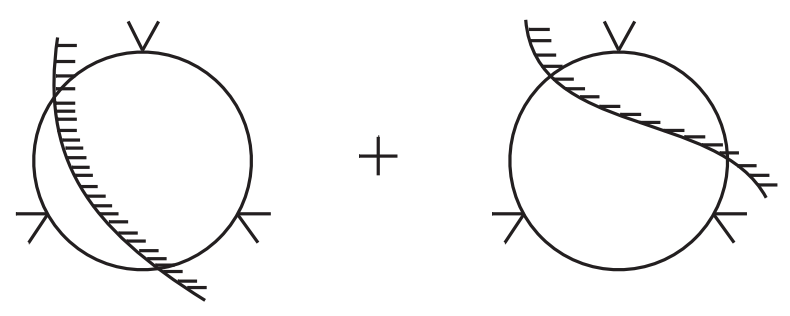

Figure 14: Cut triangle diagrams

Now, observe that all the ratios that appear here are real except the last one, which has the form

$$
\frac{1}{a-i \epsilon} \frac{1}{b-i \epsilon^{\prime}}
$$

We need to calculate the imaginary part of this expression, which can be handled by using the identity

$$
\begin{aligned}
\operatorname{Im}\left[\frac{1}{a-i \epsilon} \frac{1}{b-i \epsilon^{\prime}}\right] & =\frac{1}{2 i}\left(\frac{1}{a-i \epsilon}-\frac{1}{a+i \epsilon}\right) \frac{1}{b+i \epsilon^{\prime}}+\frac{1}{2 i}\left(\frac{1}{b-i \epsilon^{\prime}}-\frac{1}{b+i \epsilon^{\prime}}\right) \frac{1}{a-i \epsilon} \\
& =\frac{\pi \delta(a)}{b+i \epsilon^{\prime}}+\frac{\pi \delta(b)}{a-i \epsilon} .
\end{aligned}
$$

The first contribution of the last line leads to

$$
-\frac{\pi \delta\left(\omega_{1}+\omega_{3}-q^{0}\right)}{4 \omega_{1} \omega_{3}\left(m_{1}^{4}+M^{4}\right)\left(m_{3}^{4}+M^{4}\right)} \frac{M^{12}}{\left|\left(\omega_{1}-p^{0}\right)^{2}-\nu_{2}^{2}\right|^{2}} \frac{1}{\omega_{1}+\omega_{2}-p^{0}+i \epsilon_{+}^{12}} \mathcal{P} \frac{1}{\omega_{1}-\omega_{2}-p^{0}} .
$$

Now, observe that if we replace the principal value in this expression with another prescription, the difference

$$
-\frac{i \pi^{2} M^{12} \delta\left(\omega_{1}+\omega_{3}-q^{0}\right) \delta\left(\omega_{1}-\omega_{2}-p^{0}\right)}{8 \omega_{1} \omega_{2} \omega_{3}\left(m_{1}^{4}+M^{4}\right)\left(m_{2}^{4}+M^{4}\right)\left(m_{3}^{4}+M^{4}\right)}
$$

is purely imaginary. The contributions of this type cancel out from the formula for $i \mathcal{M}-i \mathcal{M}^{*}=$ $i$ Disc $\mathcal{M}$, as long as we manage to write it in a manifestly real way. We proceed by changing the prescription in a convenient way and check the cancelations in the final result (6.9).

With a new prescription, the contribution of (6.6) to $i \mathcal{M}-i \mathcal{M}^{*}$ can be turned into

$$
-\lambda^{3} \int \frac{\mathrm{d}^{D} k}{(2 \pi)^{D}} \theta\left(k^{0}\right) D_{c}\left(k^{2}, m_{1}^{2}\right) D^{*}\left((k-p)^{2}, m_{2}^{2}, \epsilon_{+}^{12}\right) \theta\left(q^{0}-k^{0}\right) D_{c}\left((k-q)^{2}, m_{3}^{2}\right) \equiv-\mathcal{C}_{1},
$$

where $\mathcal{C}_{1}$ is the first cut diagram of fig. 14, calculated with the right cut propagators [i.e. $\left.\theta\left( \pm p^{0}\right) D_{c}\left(p^{2}, m^{2}\right)\right]$, which propagate only the physical degrees of freedom. Similarly, the second contribution of $(6.5)$ gives $-\mathcal{C}_{2}$, where $\mathcal{C}_{2}$ is the second cut diagram. 
Repeating the same steps for $z_{2}$ and $z_{3}$ we find minus the other four cut diagrams $\mathcal{C}_{j}$, $j=3, \ldots 6$, which can be obtained by permuting the vertices of the cut diagrams shown in fig. 14. The total gives the correct cutting equation

$$
i \mathcal{M}-i \mathcal{M}^{*}=-\sum_{j=1}^{6} \mathcal{C}_{j} .
$$

Note that the right-hand side of this formula is manifestly real, as promised. Indeed, its imaginary part is obtained by replacing the noncut propagators with delta functions, which gives contributions evaluated on the physical poles of all three propagators. This makes them equal to analogous contributions found in the cutting equation of the standard triangle, times a real constant. Since those contributions cancel out in the case of the standard triangle, the right-hand side of (6.9) is also real.

Again, formula (6.9), which is nothing but the identity $i T-i T^{\dagger}=-T T^{\dagger}$ in the particular case of the triangle diagram, shows that no unphysical degrees of freedom propagate through the cuts, which confirms the perturbative unitarity of the LW model.

\section{Unitarity with nontrivial numerators}

So far, we have considered only theories with nonderivative vertices. For most applications, such as quantum gravity and gauge theories, it is necessary to include the case where vertices carry derivatives, which leads to nontrivial numerators in the loop integrals. We show that their presence does not change the previous results. We understand that the dimensional regularization is used, which makes it possible to apply the residue theorem even when the integral on the energy is divergent (for details on this, see the appendix of ref. [2]).

We assume that the Lagrangian is Hermitian, because this is an essential requirement for unitarity. Then the vertices, which carry an additional factor $i$, are anti-Hermitean. Denote a vertex with $n$ legs by $V_{\alpha_{1} \ldots \alpha_{n}}^{\mu_{1} \ldots \mu_{n}}(p, k)$, where $p$ and $k$ denote the external and loop momenta, respectively, $\mu_{i}$ are Lorentz indices and $\alpha_{i}$ are any other indices. We can decompose it as

$$
V_{\alpha_{1} \ldots \alpha_{n}}^{\mu_{1} \ldots \mu_{n}}(p, k)=\sum_{j} A_{\alpha_{1} \ldots \alpha_{n}}^{(j)} T_{j}^{\mu_{1} \ldots \mu_{n}}(p, k),
$$

where $T_{j}^{\mu_{1} \ldots \mu_{n}}$ are real tensor polynomials and $A_{\alpha_{1} \ldots \alpha_{n}}^{(j)}$ are constant anti-Hermitian tensors. We can focus on loop integrals with numerators made of products of tensors $T_{j}^{\mu_{1} \ldots \mu_{n}}(p, k)$.

Unitarity still holds, since the main arguments of the previous sections are determined by the locations of the poles, which do not change. For example, let us check that the ill-defined distributions cancel out. Consider formulas (4.4). If a numerator is present, we can incorporate 
it into the function $h$ that appears in the $\operatorname{sum} \operatorname{Res}\left(z_{1}\right)+\operatorname{Res}\left(z_{2}\right)$ of (5.9), so the total (5.10) is still regular. The argument is basically the same for the triangle and more complicated diagrams. Moreover, the crucial identities (5.8) and (5.6) still hold. The Hermiticity of the Lagrangian ensures (5.6) up to the effects due to $i \epsilon$, which still have the form shown on the right-hand side of (5.8). Specifically, the limit $\epsilon \rightarrow 0$ generates integrable singularities $\sim 1 / z$, where $z$ is a complex function of the integration variables that cannot vanish when the LW pinching takes place. Then, the two contributions of $\tilde{\delta}(z)$ cancel each other. In the end, the residues of the LW poles simplify in the cutting equations, so the only contributions that survive are those coming from the standard poles. It is also clear that most of these features are independent of the particular diagram that we are considering, so we expect them to hold in every diagram.

\section{Violations of unitarity in Minkowski higher-derivative theories}

The LW models are defined as nonanalytically Wick rotated Euclidean higher-derivative theories. In sections 5 and 6 we have made explicit calculations to verify that they satisfy the unitarity equation.

It is interesting to inquire whether the Minkowski versions of the same models satisfy the unitarity equation or not. By "Minkowski versions" we mean that the integrals on the energies are not performed along the integration path of fig. 1 derived in section 2, but along the real $k^{0}$ axis, as we would normally do. The integration path splits each LW pair into a pole above the path and a pole below the path. We expect that the unitarity equation is violated in this case. However, the violation is not visible at the tree level, because no energy integrals are involved: the tree cutting equations of the Minkowski models are identical to those of the LW models. Thus, it is necessary to make a one-loop calculation.

In this section we prove that the bubble diagram of the Minkowski models does not satisfy the expected cutting equation. The example we consider is a particular case where the nonlocal divergences pointed out in ref. [2] are absent.

The loop integral is still (5.1), but now, when we integrate on $k^{0}$ and close the integration path in the lower half plane, we get contributions from a different set of residues. We still have

the physical poles $z_{1}$ and $z_{2}$ of (5.2), as well as $w_{1}^{*}$ and $w_{2}^{*}$. However, we have $w_{1}^{\prime}=-\nu_{1}$ and $w_{2}^{\prime}=p^{0}-\nu_{2}$ instead of $w_{1}$ and $w_{2}$.

If the Minkowski theory were unitary, its cut diagrams would coincide with those of the LW theory, because the physical degrees of freedom are the same. Thus, if we subtract the cutting equation (4.10) of the LW theory from the one of the Minkowskian theory, the right- 


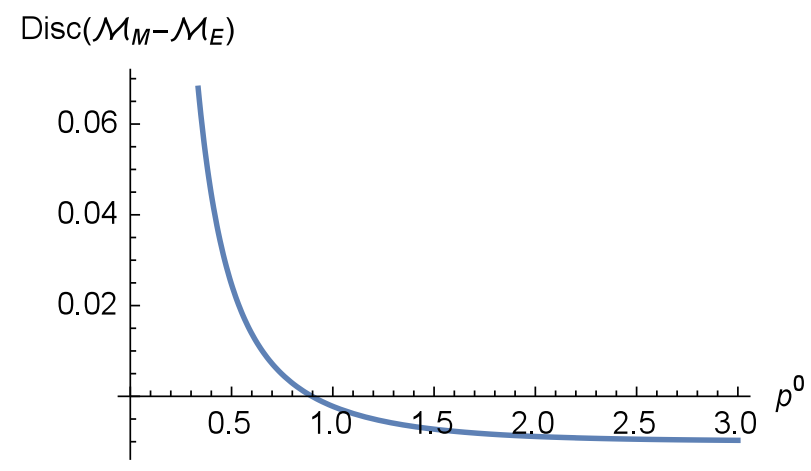

Figure 15: Difference between the Minkowski and Wick rotated Euclidean theories

hand side should give zero. We show that, instead, the discontinuity $\operatorname{Disc}\left(\mathcal{M}_{M}-\mathcal{M}_{E}\right)$ of the difference $\mathcal{M}_{M}-\mathcal{M}_{E}$ between the Minkowski amplitude $\mathcal{M}_{M}$ and the nonanalytically Wick rotated Euclidean amplitude $\mathcal{M}_{E}$ does not vanish.

Specifically, we find

$$
\operatorname{Disc}\left(\mathcal{M}_{M}-\mathcal{M}_{E}\right)=i \lambda^{2} \int \frac{\mathrm{d}^{D-1} \mathbf{k}}{(2 \pi)^{D-1}} \operatorname{Im}\left[\operatorname{Res}\left(\nu_{1}\right)+\operatorname{Res}\left(p^{0}+\nu_{2}\right)-\operatorname{Res}\left(-\nu_{1}\right)-\operatorname{Res}\left(p^{0}-\nu_{2}\right)\right] .
$$

We can simplify the calculation by choosing $\mathbf{p}=0, m_{1}=m_{2}=0$. Then we have $\nu_{1}=\nu_{2}=$ $\sqrt{\mathbf{k}^{2}+i M^{2}}, \omega_{1}=\omega_{2}=|\mathbf{k}|$. Setting $M=1$, the behavior of $\operatorname{Disc}\left(\mathcal{M}_{M}-\mathcal{M}_{E}\right)$ as a function of $p^{0}$ is nontrivial. Numerically, we find the plot of fig. 15 times $i \lambda^{2}$. This proves that the Minkowski theories violate perturbative unitarity.

\section{Conclusions}

In this paper, we have investigated the perturbative unitarity of the Lee-Wick models, formulated as nonanalytically Wick rotated Euclidean theories. We have shown that it is possible to study the cutting equations in each analytic region $\mathcal{A}_{i}$ of the complex plane, by deriving them in suitable subdomains $\mathcal{O}_{i}$ and then analytically extending the equations to the whole regions $\mathcal{A}_{i}$. The unitary cutting equations hold in each $\mathcal{A}_{i}$, with no propagation of unphysical degrees of freedom. We have made explicit computations in the cases of the bubble and triangle diagrams, but the derivations can be extended to all the one-loop diagrams. Moreover, the basic arguments do not appear to depend on the specific cases we have dealt with, so we believe that the conclusions hold for all diagrams.

On the other hand, the Minkowski versions of the same higher-derivative theories violate unitarity. In a way or another (violations of the locality of counterterms as shown in ref. [2], 
or violations of unitarity) the Minkowski higher-derivative theories are not viable. This means that, in some sense, quantum field theory prefers what we may call "Wick spacetime", i.e. the Wick rotated Euclidean space, to the Minkowski spacetime.

\section{Acknowledgments}

We are grateful to U. Aglietti and L. Bracci for useful discussions.

\section{References}

[1] G. 't Hooft and M. Veltman, One-loop divergences in the theory of gravitation, Ann. Inst. Poincaré, 20 (1974) 69;

P. van Nieuwenhuizen, On the renormalization of quantum gravitation without matter, Ann. Phys. (NY) 104 (1977) 197;

M.H. Goroff and A. Sagnotti, The ultraviolet behavior of Einstein gravity, Nucl. Phys. B 266 (1986) 709;

A. van de Ven, Two loop quantum gravity, Nucl. Phys. B 378 (1992) 309;

S. Weinberg, Ultraviolet divergences in quantum theories of gravitation, in An Einstein centenary survey, edited by S. Hawking and W. Israel (Cambridge University Press, Cambridge 1979), p. 790 .

[2] U.G. Aglietti and D. Anselmi, Inconsistency of Minkowski higher-derivative theories, Eur. Phys. J. C 77 (2017) 84, 16A2 Renormalization.com and arXiv:1612.06510 [hep-th].

[3] T.D. Lee and G.C. Wick, Negative metric and the unitarity of the S-matrix, Nucl. Phys. B 9 (1969) 209.

[4] T.D. Lee and G.C. Wick, Finite theory of quantum electrodynamics, Phys. Rev. D 2 (1970) 1033.

[5] R.E. Cutkosky, P.V Landshoff, D.I. Olive, J.C. Polkinghorne, A non-analytic S matrix, Nucl.Phys. B12 (1969) 281-300.

[6] N. Nakanishi, Lorentz noninvariance of the complex-ghost relativistic field theory, Phys. Rev. D 3, 811 (1971). 
[7] T.D. Lee, A relativistic complex pole model with indefinite metric, in Quanta: Essays in Theoretical Physics Dedicated to Gregor Wentzel (Chicago University Press, Chicago, 1970), p. 260.

[8] B. Grinstein, D. O'Connell and M.B. Wise, Causality as an emergent macroscopic phenomenon: The Lee-Wick O(N) model, Phys. Rev. D 79 (2009) 105019 and arXiv:0805.2156 [hep-th].

[9] D. Anselmi and M. Piva, A new formulation of Lee-Wick quantum field theory, J. High Energy Phys. 06 (2017) 066, 17A1 Renormalization.com and arXiv:1703.04584 [hep-th].

[10] R.E. Cutkosky, Singularities and discontinuities of Feynman amplitudes, J. Math. Phys. (NY) 1 (1960) 429;

M. Veltman, Unitarity and causality in a renormalizable field theory with unstable particles, Physica 29 (1963) 186;

G. 't Hooft, Renormalization of massless Yang-Mills fields, Nucl. Phys. B 33 (1971) 173;

G. 't Hooft, Renormalizable Lagrangians for massive Yang-Mills fields, Nucl. Phys. B 35 (1971) 167.

[11] D. Anselmi, Aspects of perturbative unitarity, Phys. Rev. D 94 (2016) 025028, 16A1 Renormalization.com and arXiv:1606.06348 [hep-th].

[12] D. Anselmi, Algebraic cutting equations, 16A3 Renormalization.com and arXiv:1612.07148 [hep-th].

[13] B. Grinstein, D. O'Connell, and M.B. Wise, The Lee-Wick standard model, Phys. Rev. D77 (2008) 025012 and arXiv:0704.1845 [hep-ph];

C.D. Carone and R.F. Lebed, Minimal Lee-Wick extension of the standard model, Phys. Lett. B668 (2008) 221 and arXiv:0806.4555 [hep-ph];

J.R. Espinosa and B. Grinstein, Ultraviolet properties of the Higgs sector in the Lee-Wick standard model, Phys. Rev. D83 (2011) 075019 and arXiv:1101.5538 [hep-ph];

C.D. Carone and R.F. Lebed, A higher-derivative Lee-Wick standard model, JHEP 0901 (2009) 043 and arXiv:0811.4150 [hep-ph].

[14] B. Grinstein and D. O'Connell, One-Loop Renormalization of Lee-Wick Gauge Theory, Phys. Rev. D78 (2008) 105005 and arXiv:0801.4034 [hep-ph];

C. D. Carone, Higher-derivative Lee-Wick unification, Phys. Lett. B677 (2009) 306, 
and arXiv:0904.2359 [hep-ph].

[15] E. Tomboulis, 1/N expansion and renormalization in quantum gravity, Phys. Lett. B 70 (1977) 361;

E. Tomboulis, Renormalizability and asymptotic freedom in quantum gravity, Phys. Lett. B 97 (1980) 77;

L. Modesto, Super-renormalizable or finite Lee-Wick quantum gravity, Nucl. Phys. B909 (2016) 584 and arXiv:1602.02421 [hep-th].

[16] I. Shapiro and L. Modesto, Superrenormalizable quantum gravity with complex ghosts, Phys. Lett. B755 (2016) 279-284 and arXiv:1512.07600 [hep-th].

[17] See, for example, M. E. Peskin, D. V. Schroeder, An introduction to quantum field theory (Reading, USA, Addison-Wesley, 1995), Chapter 7, section 3. 